\title{
WestVirginiaUniversity
}

THE RESEARCH REPOSITORY @ WVU

Graduate Theses, Dissertations, and Problem Reports

2014

\section{Optimal Scheduling of Power Plant Maintenance with Gas Portfolio}

\author{
Yixin Du \\ West Virginia University
}

Follow this and additional works at: https://researchrepository.wvu.edu/etd

\section{Recommended Citation}

Du, Yixin, "Optimal Scheduling of Power Plant Maintenance with Gas Portfolio" (2014). Graduate Theses, Dissertations, and Problem Reports. 283.

https://researchrepository.wvu.edu/etd/283

This Thesis is protected by copyright and/or related rights. It has been brought to you by the The Research Repository @ WVU with permission from the rights-holder(s). You are free to use this Thesis in any way that is permitted by the copyright and related rights legislation that applies to your use. For other uses you must obtain permission from the rights-holder(s) directly, unless additional rights are indicated by a Creative Commons license in the record and/ or on the work itself. This Thesis has been accepted for inclusion in WVU Graduate Theses, Dissertations, and Problem Reports collection by an authorized administrator of The Research Repository @ WVU. For more information, please contact researchrepository@mail.wvu.edu. 


\title{
Optimal Scheduling of Power Plant Maintenance with Gas Portfolio
}

\author{
by \\ Yixin Du \\ Thesis submitted to the \\ College of Engineering and Mineral Resources \\ at West Virginia University \\ in partial fulfillment of the requirements \\ for the degree of \\ Master of Science \\ in \\ Industrial Engineering \\ Feng Yang, Ph.D. \\ Wafik H. Iskander, Ph.D. \\ Qipeng Phil Zheng, Ph.D., Chair \\ Department of Industrial and System Management Engineering \\ Morgantown, West Virginia \\ 2014
}

Keywords: Power Plan Maintenance Scheduling, Gas Portfolio, Mathematical Modeling

Copyright 2014 Yixin Du 


\author{
Abstract \\ Optimal Scheduling of Power Plant Maintenance with Gas Portfolio \\ by \\ Yixin Du \\ Master of Science in Industrial Engineering \\ West Virginia University \\ Qipeng Phil Zheng, Ph.D., Chair
}

Power plant maintenance scheduling aims at defining the sequence of preventive maintenance outages of each unit over the planning time horizon so that the overall costs are minimized and all the constraints are satisfied. Optimization in maintenance scheduling could reduce greenhouse gases and help meeting the surging global energy demand. Maintenance scheduling with gas portfolio could not only cut down the cost for purchasing gas and performing maintenance in the power plant, they could also stabilize the gas network and electricity grid. The goal of this research is to build an applicable mathematical model for optimizing the maintenance scheduling as well as gas purchasing strategy. Firstly, a novel stochastic mathematical model is proposed for making production and maintenance decisions. Then the reformulation of the original model is introduced in order to obtain a tighter convex hull. Thirdly, the reformulated model is decomposed by Branch-and-Price algorithm for solving large-scale problems. Finally, the computational results are compared and future research is proposed. This research helps reduce the risks and costs in the power generation when decision making is needed by managers, investors and traders. 


\section{Acknowledgements}

I would like to firstly thank my committee chair and advisor, Dr. Qipeng Phil Zheng, for giving me the opportunity to work with him and his students. This thesis would not be possible without his constant guidance and support.

I would also like to thank Dr. Wafik H. Iskander and Dr. Feng Yang for being on my committee. I have been fortunate to have had the opportunity to ask for suggestions with all of my committee members, and their help has been essential to this project.

Next, I would also like to thank the students in Dr. Zheng's research group with whom I've had the pleasure of working alongside. In particular, I would like to thank my colleague Yuping Huang, who has been a great help to me over the last two years.

Finally, I would like to express my gratitude to my family. Their support seemingly has no limit, and has been much appreciated throughout my life.

This work has been in part supported by the National Science Foundation under grant ECCS-1232168. 


\section{Contents}

Acknowledgements $\quad$ iii

List of Figures $\quad$ vi

List of Tables $\quad$ vii

1 Introduction to Power Plant Maintenance Scheduling $\quad 1$

2 Literature Review of Maintenance Scheduling Models $\quad 7$

2.1 Decisions . . . . . . . . . . . . . . . . . . . 7

2.2 Objectives . . . . . . . . . . . . . . . . . . . 8

2.2.1 Least-cost based criterion . . . . . . . . . . . . . . 8

2.2 .2 Reliability based criterion . . . . . . . . . . . . . . . . . . 9

2.3 Constraints . . . . . . . . . . . . . . . . . . . 11

2.3.1 Maintenance constraints . . . . . . . . . . . . . . . . 11

2.3.2 Generation constraints . . . . . . . . . . . . . . . . . 12

2.4 Deterministic Model . . . . . . . . . . . . . . . . . . . . . 15

2.5 Stochastic Model . . . . . . . . . . . . . . . . . . . . . . 17

3 Power Plant Maintenance Considering Fuel Contracts 22

3.1 Problem Statement . . . . . . . . . . . . . . . . . . . . 22

3.1.1 Maintenance Scheduling with Gas Portfolio . . . . . . . . . . . . . . . 22

3.1.2 The Assumptions . . . . . . . . . . . . . . . . . . . 25

3.2 Stochastic Model . . . . . . . . . . . . . . . . . . . 25

3.2.1 Introduction to Stochastic Programming . . . . . . . . . . . . 25

3.2 .2 General Nomenclature . . . . . . . . . . . . . . . . . 27

3.2 .3 Objective Function . . . . . . . . . . . . . . . . . . . 27

3.2 .4 Constraints . . . . . . . . . . . . . . . . . 29

3.2 .5 Optimization Model . . . . . . . . . . . . . . . . 32

3.3 Model Reformulation . . . . . . . . . . . . . . . . . . . . . . . 32

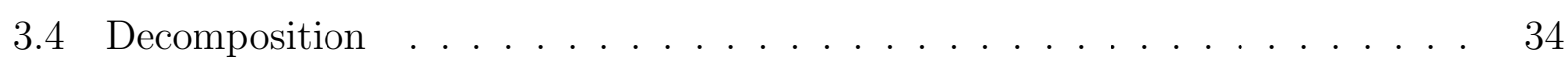

3.5 Computational Results . . . . . . . . . . . . . . . . . . . 37

3.5.1 Analyze the Solution of the Original Model . . . . . . . . . . . . . . 37 
3.5.2 Compare the Solution and Computational Time of the Original and Reformulated Model . . . . . . . . . . . . . . . . 38

3.5.3 Solution of the Decomposed Model . . . . . . . . . . . . . . . . 42

3.6 Conclusions and Future Research . . . . . . . . . . . . . . . . . . 42

$\begin{array}{ll}\text { References } & 47\end{array}$ 


\section{List of Figures}

1.1 Primary energy consumption by sector in U.S, 2011 (Sources: EIA data) . . 2

1.2 Growth in world real GDP and growth in world energy use (Sources: World Bank data) . . . . . . . . . . . . . . . . . . . . . 3

3.1 Gas transfer direction . . . . . . . . . . . . . . . . . . . . . . . . . . 24

3.2 Multistage decision process . . . . . . . . . . . . . . . . . 26

3.3 Scenario tree . . . . . . . . . . . . . . . . . . 27

3.4 Reformulation technique . . . . . . . . . . . . . . . . 33

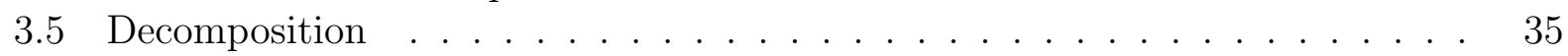

3.6 Compare computational time when $t=6 \ldots \ldots \ldots \ldots$. . . . . . . . . . . . . . . . . . . . . .

3.7 Compare computational time when $t=9 \ldots \ldots \ldots$. . . . . . . . . 41

3.8 Compare computational time when $t=12 \ldots \ldots \ldots$. . . . . . . . 41 


\section{List of Tables}

2.1 Summary of decisions . . . . . . . . . . . . . . . . . . . . . . . . . . . . . . . . . .

2.2 Summary of objectives . . . . . . . . . . . . . . . . . . . . 10

2.3 Summary of constraints . . . . . . . . . . . . . . . . . 14

2.4 Summary of assumptions in deterministic model . . . . . . . . . . . . . . . 15

2.5 Summary of assumptions in stochastic model . . . . . . . . . . . . . . . 21

3.1 Maintenance factors . . . . . . . . . . . . . . . . . . . . . 25

3.2 Indices . . . . . . . . . . . . . . . . . . . . . . 27

3.3 Variables . . . . . . . . . . . . . . . . . . . . . . . . 28

3.4 Parameters . . . . . . . . . . . . . . . . . . . . . . 28

3.5 Input parameters of $\operatorname{prices}(\$ /$ MMBTU $) \ldots \ldots \ldots \ldots$

3.6 Other parameters . . . . . . . . . . . . . . . . . . 37

3.7 Objective value of the original model using parameters in Table 3.5 \& 3.6 . . 38

3.8 Solution of the original model using parameters in Table $3.5 \& 3.6$. . . . . . 38

3.9 Objective value of the reformulated model using parameters in Table 3.5 \& 3.639

3.10 Solution of the reformulated model using parameters in Table 3.5 \& 3.6 . . . 39

3.11 Solution of $W_{z_{n}}$ of decomposed model, Part $1 \ldots \ldots$. . . . . . . . 45

3.12 Solution of $W_{z_{n}}$ of decomposed model, Part $2 \ldots \ldots \ldots$ 


\section{Chapter 1}

\section{Introduction to Power Plant Maintenance Scheduling}

Energy is a crucial element in our daily life. The primary energy sources include petroleum, natural gas, coal, renewable energy, and nuclear energy. These energy sources are converted and consumed for generating electricity, transportation, industry, commercial, and residential buildings use. The primary energy consumption by sector in 2011 is shown in Figure 1.1 [1]. Power generation is the largest sector in the United States which consumed nearly 40 percent of primary energy sources.

Electricity is a secondary energy source which is converted from the primary energy sources. In 2011, nearly 46 percent of electricity was generated from coal. Most power plants use steam turbines which are connected with generators. A steam turbine converts kinetic energy to mechanical energy. The generator connected with steam turbine converts mechanical energy to electrical energy.

According to U.S. Energy information administration (EIA), the average of energy expenditures as a share of gross domestic product (GDP)in the U.S. was 8.8 percent from 1970 to 2010 [2]. Figure 1.2 shows the relationship between World GDP growth and World energy use from 1971-2010. Prior to 2008, the growth in World GDP was faster than the growth in World energy use. From 2008-2009, both of them have a big drop because of financial crisis. However, the growth rate of energy use exceeded the GDP in 2009. Unlike the growth of GDP, the growth of energy use fluctuated in history. The overall trend of both of them is 


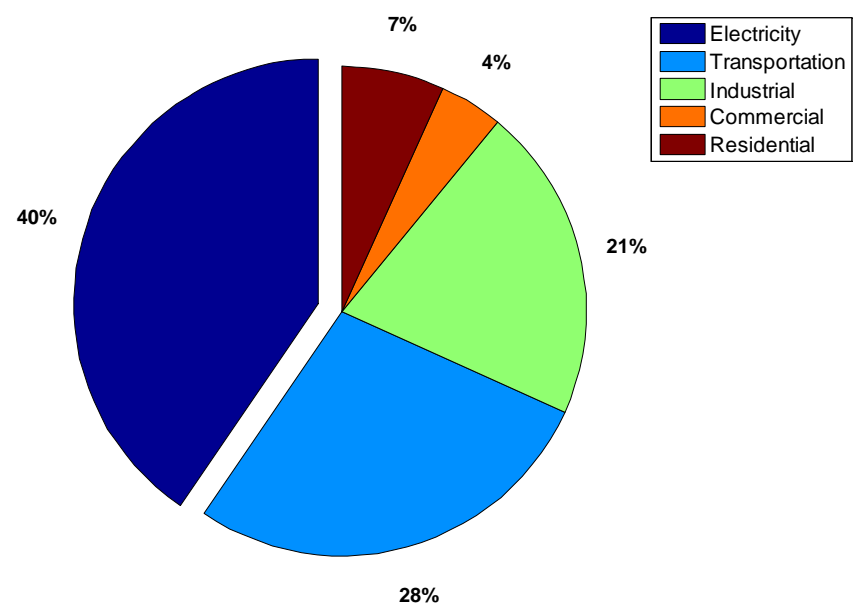

Figure 1.1: Primary energy consumption by sector in U.S, 2011 (Sources: EIA data)

increasing. We can conclude that the growth of energy use could boom the World GDP. It is a factor which indicates the trend of the growth of GDP.

According to EIA, at the beginning of the 20th century, there were 4000 electric utilities which were operated individually. Local customers were serviced from nearby power plants which were connected with low-voltage transmission lines. With the growing demand for electricity during the last 40 years, it is much more efficient to implement larger generators and connect power plants in different areas with high-voltage transmission lines. In the way of implementing the interconnected system, the operation cost for power plant has been significantly reduced and reliability could be promised.

The average power plant maintenance expenses for major U.S. investor-owned electric utilities, such as nuclear, fossil steam, and hydro-electric utilities, have kept increasing from 2001 to 2011 [3]. Large-scale system has further complicated the maintenance scheduling problem. Even a small improvement of the maintenance scheduling strategy could reduce the maintenance cost significantly. Thus a lot of decision makers and researchers have focused on maintenance scheduling problem, finding a way to reduce operational cost and ensure system reliability.

Maintenance includes a variety of topics, such as corrective maintenance, planned maintenance, condition-based maintenance, preventive maintenance, and so on. Corrective maintenance is performed when failure happens in the system [4]. Planned maintenance is a 


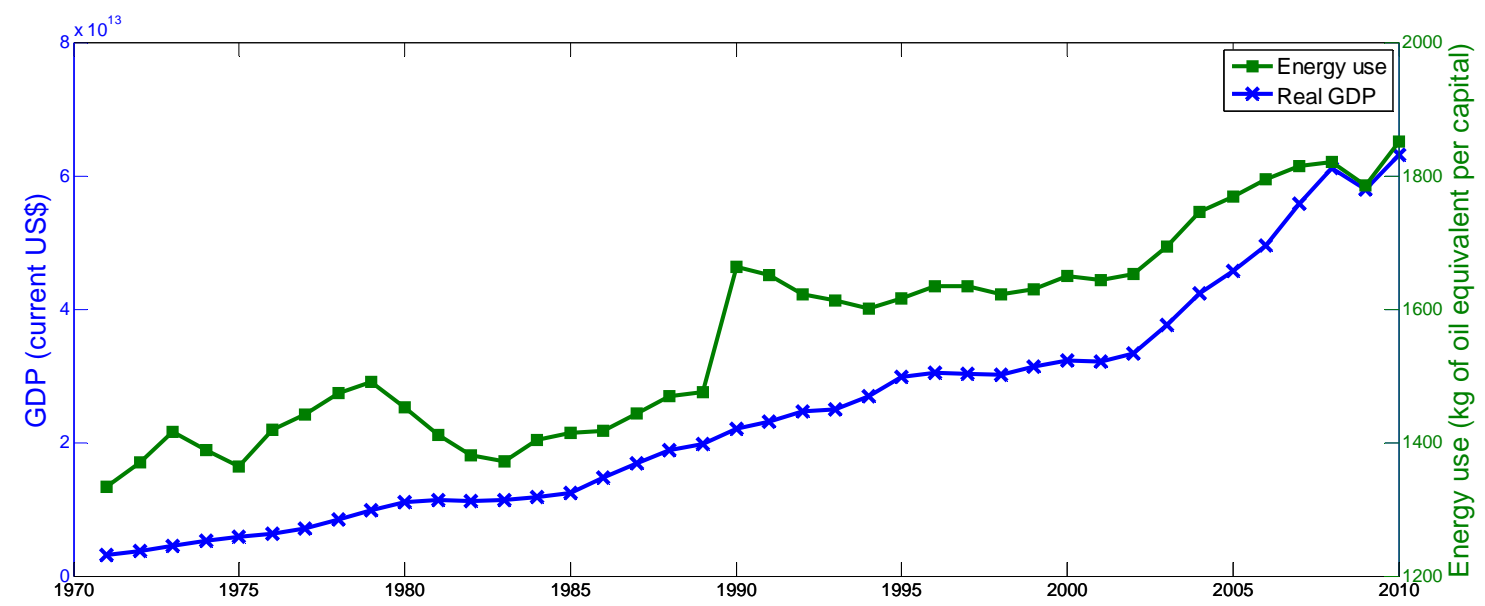

Figure 1.2: Growth in world real GDP and growth in world energy use (Sources: World Bank data)

scheduled maintenance to an item. Conditional-based maintenance is performed when need arises. Condition-based maintenance and planned maintenance comprise preventive maintenance. In power plants, we mainly focus on the preventive maintenance scheduling.

Before we discuss maintenance scheduling, a brief introduction is given to electricity market. The objective of an electricity market is to ensure a secure and economical operation. This could be realized by implementing a restructured power system. Restructuring could reduce the cost of electricity, and enhance the system security. There are two components of a restructured power system: market operators and market participants [5]. A market operator is independent of the market participants, e.g. the independent system operator (ISO). The objective of ISO is to coordinate the maintenance scheduling among market participants and maintain the system security. The key market participants are generating companies (GENCOs) and transmitting companies (TRANSCOs). Other market participants include distributing companies (DISCOs), customers, and so on. GENCOs not only manage and maintain generating plants, but also coordinate the maintenance of generating units with ISO by performing maintenance which is approved by ISO. Their goal is to maximize profits. TRANSCOs manage and maintain the transmission system, in order to provide a reliable electricity system. In order to better understand the maintenance scheduling problem, we explain some basic concepts: 
- Unit: Unit includes turbines, boilers, generators, transmission lines, and so on. An outage of a unit will result in a loss of capacity. It also requires manpower and resource from a limited pool to do the maintenance. A unit must be scheduled for maintenance after being used for a given number of time intervals.

- Time horizon: The time period which is considered for maintenance scheduling.

- Maintenance cost: Cost of the maintenance of a unit in an outage, such as labor cost, purchasing new parts for replacement, and so on.

- Generation cost: Cost to generate electricity.

- Loss-of-load-probability (LOLP): The probability that the system generation available is less than the system load.

- Forced outage: A unit will not be able to provide service when required. Forced outage time is the time during which the unit is in forced outage.

The maintenance scheduling aims at defining the sequence of preventive maintenance outages of each unit over the planning time horizon so that the maintenance cost and generation cost are minimized, the resource constraint, system reliability constraint, and a number of other constraints are satisfied.

Typically, the time horizon of maintenance scheduling is categorized into three classes: short-term maintenance scheduling (STMS), mid-term maintenance scheduling (MTMS), and long-term maintenance scheduling (LTMS). The time scales which have been used are as follows:

- STMS: a few weeks prior to the start of maintenance;

- MTMS: a few months to one year prior to the start of maintenance;

- LTMS: more than one year prior to the start of maintenance.

Except for the maintenance scheduling problem, unit commitment is a critical problem for electricity generation. The unit commitment problem is to minimize the generation cost by finding an optimal commitment scheduling of generation units [6]. Several issues within 
the maintenance scheduling have also been considered in the unit commitment, such as: reliability, generation capacity, transmission constraints, stochastic demand [7]. Many techniques, such as Lagrangian relaxation [8], branch-and-bound [9], and Benders decomposition techniques are used to solve the unit commitment problem [10] [11].

We classify the maintenance scheduling goals into three points of view: equipment, power plant, and power market.

From the equipment point of view: Preventive maintenance, including check-ups, repair, and parts replacement, performed to keep unit failures from happening. A timely maintenance could bring the failed units into good working condition, reduce the happening of outages, slow the degradation process and extend the equipment life [12]. If an outage takes place too late, the forced outage rate will increase and repair will become more expensive. In order to increase the availability, all the units are recommended to be maintained in a timely manner.

From the power plant point of view: The maintenance goals are minimizing maintenance costs and generation costs, reducing LOLP, and ensuring the system reliability. The reliability of power systems and operational costs are highly associated with the maintenance of generating units. Tightening reserve and avoiding unnecessary maintenance could increase system reliability and decrease the operation cost.

From the market point of view: a well-designed maintenance schedule could minimize the cost of generating electricity so that the social welfare can be promised. It could also prevent blackout from happening.

The maintenance scheduling is a large-scale constrained optimization problem. Some key elements need to be considered, such as fuel supply, market price for electricity, forced outages, load, and resource availability. Also, there are dozens to hundreds of units in modern power plants, which means that dozens to hundreds of variables exist. When we consider time increments as well, the number of feasible solutions to the problem is extremely high, which is called the curse of dimensionality.

The complexities of the maintenance scheduling are a result of the following aspects of power system:

- Supply must equal to demand at every second, in other words, they happen simulta- 
neously. This is the most important feature in power system, if supply is less than demand, load shedding may occur and then customers' needs could not be satisfied.

- In addition to keep supply equal to demand, a sufficient level of reserve energy is required to ensure reliability. The extra amount of energy is produced to deal with the demand fluctuation. Meanwhile, it compensates the shortage in electricity generation when forced outages happen.

- GENCO and TRANSCO are connected to the power pool. Not only should the maintenance policy for each GENCO be considered, the maintenance coordination from the pool level should also be concerned. As we mentioned above, modern restructured power system is comprised with many GENCOs and TRANSCOs, if one of them loses service, the rest will be affected. 


\section{Chapter 2}

\section{Literature Review of Maintenance Scheduling Models}

In this chapter, we will introduce different decision variables, objectives, and constraints of maintenance models of previous literature. Then we will analyze and compare the modeling techniques, and the effectiveness of deterministic models and stochastic models.

\subsection{Decisions}

The most important set of decisions represent the status of units, such as maintenance, start-up, shut-down, and online status. These decision variables define the time period of occurrences of maintenance, online/offline, start up and shut down of generators.

Another set of decision variables represent the decisions involved in electricity generating process, such as the output of generator, expected energy purchased from outside resources, fuel allocation, and expected energy not served (EPNS).

Table 2.1 lists different variables which have been used in previous literature. $x_{i, t}$ is used to represent maintenance starting time, it defines the status and time period of maintenance of a unit. $p_{i, t}$ is the output of generator which is closely related with the maintenance, when the number of maintenance increases, the other one will decrease. $z_{i, t}$ and $y_{i, t}$ are used when one consider unit commitment problem and maintenance scheduling problem simultaneously. 


\begin{tabular}{|c|c|c|c|}
\hline Type & Decisions & Explanation & References \\
\hline$x_{i, t}$ & Binary & $\begin{array}{l}\text { Maintenance status of unit } \mathrm{i} \text { in pe- } \\
\text { riod } t, 0 \text { if a unit (i) is on mainte- } \\
\text { nance in period } t \text {, otherwise } 1 .\end{array}$ & $\begin{array}{l}{[13],[14],[15],[16],[16],[17],} \\
{[18],[19],[20],[21],[22],[23],} \\
{[24],[25],[26],[27],[28],[29],} \\
\text { [30] }\end{array}$ \\
\hline$y_{i, t}$ & Binary & $\begin{array}{l}\text { Start up status of units, } 1 \text { if a u- } \\
\text { nit starts up at the beginning of } \\
\text { period t, otherwise } 0\end{array}$ & {$[31],[16]$} \\
\hline$z_{i, t}$ & Binary & $\begin{array}{l}\text { Online status of units of generator } \\
i, 1 \text { if a unit is connected in period } \\
\text { t, otherwise } 0\end{array}$ & {$[16]$} \\
\hline$p_{i, t}$ & Continuous & Output of unit $\mathrm{i}$ in period $\mathrm{t}$ & $\begin{array}{l}\text { [13], [31], [14], [15], [16], [16], } \\
{[18],[29],[20],[32],[21]}\end{array}$ \\
\hline$k_{t}$ & Continuous & $\begin{array}{l}\text { Expected cost of energy purchased } \\
\text { from outside resources at period t }\end{array}$ & {$[33],[16],[26]$} \\
\hline$\phi_{i, t}$ & Continuous & $\begin{array}{l}\text { Amount of fuel allocated to unit i } \\
\text { during period } t\end{array}$ & {$[14]$} \\
\hline$\gamma_{t}$ & Continuous & $\begin{array}{l}\text { Expected energy not served (EP- } \\
\text { NS) in period t }\end{array}$ & {$[15]$} \\
\hline
\end{tabular}

Table 2.1: Summary of decisions

\subsection{Objectives}

In the maintenance scheduling, a key requirement is selected as the objective criterion, other requirements are formulated into constraints. There are two kinds of general objective criteria: least-cost based criterion and reliability based criterion.

\subsubsection{Least-cost based criterion}

Costs, including maintenance cost of generating utilities and transmission lines, generation cost, penalty cost for purchasing energy from nearby power plants when the demand could not be satisfied, are the main motivation considered in previous literature.

Least-cost objective criterion chooses one of the costs or some combinations of the costs summarized above as the objective.

1. Maintenance cost of unit $i$ : $C\left(1-x_{i, t}\right)$, where $C$ is constant of performing maintenance. 
2. Generation cost of generator $i$ in period $t: F_{i} U_{t} p_{i, t}$, where $F_{i}$ is the electric energy cost for generator $i(\$ / \mathrm{MWh}), U_{t}$ is the duration of period $t(\mathrm{~h})$.

3. Penalty cost for purchasing energy from other plants in period $t: k_{t}(\$)$, when demand cannot be satisfied, electricity must be purchased from other plants, typically the cost for purchasing electricity is more expensive than the electricity generated by power plants themselves.

Escudero [18] aimed to minimize the generation cost over the planning horizon, subject to four types of constraints: one-time maintenance, maintenance and connection constraint, generation capacity and demand constraint. In order to reduced the computational difficulty caused by binary variables in the non-linear problem, the last three constraints are relaxed by replacing them with some continuous constraints suggested by Biggs [34]. After transforming the original problem into a non-linear problem, it is solved through constrained non-linear programming algorithm.

\subsubsection{Reliability based criterion}

Krishnasamy et al. [35] developed a risk-based maintenance (RBM) strategy by applying four steps: identification of the scope, risk assessment, risk evaluation, and maintenance planning. Particularly, the first procedure identifies the components within the power plants, defines relationship of components in the systems. The risk assessment procedure calculates the probability of failure, and the result of each failure is analysed. The third procedure determines whether the risk of each failure scenario is acceptable or not. If it is not acceptable, the last procedure redesigns the scenario and tries to reduce the risk to an acceptable level.

Carazas and Souza [36] developed a new risk-based decision making method for maintenance policy selection. The analyst can predict the future equipment reliability based on each maintenance policy, evaluate the equipment failure consequences, and calculate the maintenance cost. Once the above procedures are completed, a decision tree is used to select the maintenance procedure which minimizes failure risk and failure cost. 


\begin{tabular}{|l|l|c|}
\hline Objectives & Examples & References \\
\hline Least cost & Minimize maintenance cost & {$[24],[27],[38],[22]$} \\
\hline Least cost & $\begin{array}{l}\text { Minimize maintenance cost plus penalty } \\
\text { cost }\end{array}$ & {$[30]$} \\
\hline Least cost & Minimize generation cost & {$[31],[28],[19]$} \\
\hline Least cost & Minimize generation cost plus start-up cost & {$[13],[17],[26],[29],[20]$} \\
\hline Least cost & $\begin{array}{l}\text { Minimize maintenance cost plus genera- } \\
\text { tion cost }\end{array}$ & {$[16],[32]$} \\
\hline Least cost & $\begin{array}{l}\text { Minimize maintenance cost plus genera- } \\
\text { tion cost plus penalty cost }\end{array}$ & {$[14],[21]$} \\
\hline Least cost & $\begin{array}{l}\text { Maximize income minus } \text { (generation cost } \\
\text { plus maintenance cost plus shut down cost) }\end{array}$ & {$[15]$} \\
\hline Least cost & $\begin{array}{l}\text { Minimize maintenance cost plus genera- } \\
\text { tion cost plus shut down cost plus start-up } \\
\text { cost plus penalty cost }\end{array}$ & $\begin{array}{l}\text { Minimize generation cost plus penalty cost } \\
\text { plus EPNS }\end{array}$ \\
\hline $\begin{array}{l}\text { Least cost } \\
\text { Reliability based } \\
\text { criterion }\end{array}$ & $\begin{array}{l}\text { Minimize the risk penalty factor subject to } \\
\text { maintaining adequate level of reliability }\end{array}$ & {$[35],[36],[21]$} \\
\hline $\begin{array}{l}\text { Reliability based } \\
\text { criterion }\end{array}$ & $\begin{array}{l}\text { Minimize the sum of squared reserve gen- } \\
\text { eration }\end{array}$ & {$[37],[40],[41],[42],[43]$} \\
\hline $\begin{array}{l}\text { Reliability based } \\
\text { criterion }\end{array}$ & $\begin{array}{l}\text { Maximize reliability index which is the net } \\
\text { reserve divided by the gross reserve in pe- } \\
\text { riod t }\end{array}$ & {$[16]$} \\
\hline
\end{tabular}

Table 2.2: Summary of objectives

Nayak et al. [37] used the levelized reserve method to evaluate the reliability. The objective function is represented by minimizing the sum of squares of reserve generation considering statutory safety regulations. A Genetic Algorithm is used to solve the problem. The reliability is represented by Loss of Load Probability (LOLP), and load forecasting is performed to evaluate the reliability.

Conejo et al. [16] explored the coordination between ISO and producers. Two models are introduced accordingly: maximizing reliability for ISO and maximizing profit for producers. In the plan for ISO, the target is to maximize the reliability index which is net reserve divided by gross reserve along the time horizon. A detailed seven-step procedure is introduced to achieve a generation maintenance plan that not only achieves a sufficient level of safety, but also maximizes producer's profit. Table 2.2 summarizes the objectives in previous works. 


\subsection{Constraints}

Here we classify maintenance scheduling constraints into two sets: Maintenance constraints and Generation constraints.

Maintenance constraints specify the starting time and sequence of maintenance outages, and point out the amount of resources required by the outages, such as maintenance duration, allowed/not allowed periods of time, non-stop maintenance, maintenance priority, and so on.

Generation constraints are applied to ensure that the impact of maintenance outages on the system is within an acceptable level. In other words, maintenance outages should not affect generating required electricity.

In the following subsection, we discuss the maintenance constraints and generation constraints which have been used in previous works.

\subsubsection{Maintenance constraints}

1. Maintenance duration: (1) ensures that each unit is maintained for a required number of periods:

$$
\sum_{t=1}^{T}\left(1-x_{i, t}\right)=D_{i}, \quad \forall i \in Q,
$$

where $D_{i}$ is the maintenance duration of unit $i, T$ is the number of periods to be considered, $Q$ is the set of units.

If the maintenance duration of unit $i$ is 2 , and the available maintenance periods is from 1 to 3 , shown as $x_{i, 1}+x_{i, 2}+x_{i, 3}=2$, the maintenance outage then could start from period 1 , and end at period 2, or start from period 2, and end at period 3.

2. Not allowed/allowed periods of time: Maintenance outages could only happen within the allowed period of time of one window,

$$
\begin{aligned}
& x_{i, t}=1, \quad t<T_{i}^{\text {min }} \text { and } t>t_{T}^{\max }, \quad \forall i \in Q, \forall t \in T, \\
& x_{i, t} \in\{0,1\}, \quad T_{i}^{\text {min }} \leq t \leq T_{i}^{\text {max }} . \quad \forall i \in Q, \quad \forall t \in T,
\end{aligned}
$$


where $T_{i}^{\min }$ is the earliest maintenance period of unit $i, T_{i}^{\max }$ is the latest maintenance period of unit $i$.

3. Resource constraint: Constraint (2.3) indicates the maximum number of maintenance which could happen simultaneously due to the manpower and other resource limits,

$$
\sum_{i}^{Q}\left(1-x_{i, t}\right) \leq N_{t}, \quad \forall t \in T
$$

where $N_{t}$ is the maximum number of maintenance outages that could happen in period $t$.

4. Exclusiveness: If it is required that maintenance for $i$ and $j$ could not happen simultaneously, then the following constraint must be held. The mathematic formulation specifies in the same time period $t$ that the summation of $x_{i, t}$ and $x_{j, t}$ should be less or equal to 1 :

$$
x_{i, t}+x_{j, t} \leq 1, \quad \forall t \in T
$$

\subsubsection{Generation constraints}

1. Power generation limit: Each generating unit is limited by its minimum and maximum capacity:

$$
z_{i, t} P_{i, t}^{\min } \leq p_{i, t} \leq z_{i, t} P_{i, t}^{\max }, \quad \forall i \in Q, \forall t \in T
$$

where $P_{i, t}^{\max }$ is the maximum power generation capacity for generator $i(\mathrm{MW})$ in period $t, P_{i, t}^{\min }$ is the minimum power generation capacity for generator $i(\mathrm{MW})$ in period $t$.

2. Demand: Power demand needs to be satisfied in each period:

$$
\sum_{i}^{Q} p_{i, t}=P D_{t}, \quad \forall t \in T,
$$

where $P D_{t}$ is the power demand in period $t(\mathrm{MW})$.

3. Reserve: In order to ensure that the total available power is greater than demand when contingency happens, a sufficient amount of power reserve must be ensured. 
This constraint could prevent power shortages for serving customers when some units are randomly in outages:

$$
\sum_{i}^{Q} z_{i, t} P_{i, t}^{\max } \geq P D_{t}+R_{t}, \quad \forall t \in T,
$$

where $R_{t}$ is the reserved power which ensures reliability in period $t(\mathrm{MW})$.

4. Start-up logic: Constraint (2.8) considers the logic of unit status between start-up and connection:

$$
z_{i, t}-z_{i, t-1} \leq y_{i, t}, \quad \forall i \in Q, \forall t \in T
$$

where $y_{i, t}$ equals to 1 if generator $i$ starts at the beginning of period $t$, otherwise 0 .

5. Maintenance and connection logic: Constraint (2.9) enforces that a unit cannot be online and on maintenance at the same time:

$$
x_{i, t}+z_{i, t} \leq 1, \quad \forall i \in Q, \forall t \in T
$$

6. Emission and fuel: An upper bound of electricity generation (MWh) is imposed to reduce the environment pollution, and make sure that the fuel constraint is not violated:

$$
\sum_{t}^{T} p_{i, t} \leq E_{i, t}, \quad \forall i \in Q
$$

where $E_{i, t}$ is the maximum amount of energy (MWh) to be produced by unit $i$ in period $t$.

For example, coal is the primary resource which has been used to generate electricity. Coal combustion is the top source of carbon dioxide emissions, which is the primary cause of global warming (Zheng et al. [44]). Taking into account the environmental issues is also critical in selecting maintenance scheduling.

7. Transmission network: The transmission line flow should not exceed the maximum transmission line capacity.

$$
f_{t} \leq F_{t}^{\max }, \quad \forall t \in T
$$




\begin{tabular}{|l|l|}
\hline Constraints & References \\
\hline Maintenance duration $(2.1)$ & {$[23],[31],[14],[15],[16],[16],[26],[27]$,} \\
& {$[39],[19],[21],[22]$} \\
\hline Not allowed/allowed periods of time & {$[23],[24],[13],[16],[17],[25],[39],[29]$,} \\
$(2.2 a),(2.2 b)$ & {$[22],[30]$} \\
\hline Resource $(2.3)$ & {$[23],[13],[31],[14],[15],[16],[17],[25]$,} \\
& {$[27],[28],[39],[29],[21],[22],[30]$} \\
\hline Exclusiveness (2.4) & {$[31],[14],[15],[16],[26],[28],[30]$} \\
\hline Power generation limit (2.5) $[23],[24],[13],[31],[16],[17],[26],[18]$, \\
\hline Demand (2.6) & {$[19],[21],[22],[30]$} \\
\hline Reserve (2.7) & {$[24],[13],[31],[26],[18],[19],[40],[29]$,} \\
\hline Start-up logic (2.8) & {$[20],[21],[22],[30]$} \\
\hline Maintenance and connection logic (2.9) & {$[31],[16],[17],[26],[28],[22]$} \\
\hline Emission and fuel limits $(2.10)$ & {$[31],[16]$} \\
\hline Transmission limit $(2.11)$ & {$[31],[16],[22]$} \\
\hline Expected energy not served (2.12) & {$[24],[13],[19],[40],[20],[21],[22],[30]$} \\
\hline
\end{tabular}

Table 2.3: Summary of constraints

where $f_{t}$ is the transmission flow in period $t, F_{t}^{\max }$ is the maximum transmission line capacity.

8. Expected energy not served: The expected energy not served in period $t$ should be within an acceptable level.

$$
\gamma_{t} \leq \xi_{t}, \quad \forall t \in T
$$

where $\xi_{t}$ is the acceptable level of EPNS in period $t$.

Table 2.3 is a summary of constraints which have been used in the maintenance scheduling models. We can conclude that maintenance duration, one-time maintenance, not allowed/allowed periods of time, resource, power generation limit, and capacity are essential constraints. Separation between consecutive maintenance outages, overlap in maintenance, start-up logic, maintenance and connection logic, and emission and fuel limits constraints are barely used. 


\begin{tabular}{|l|l|}
\hline Assumptions & References \\
\hline Power demand is known & {$[18],[29],[26],[19],[45],[20]$,} \\
& {$[16]$} \\
\hline Prices of fuel, energy, and ancillary services & {$[18],[26],[19],[29],[45],[20]$,} \\
are known & {$[16]$} \\
\hline Forced outage rate is 0 & {$[18],[26],[19],[29],[45],[20]$,} \\
& {$[16]$} \\
\hline
\end{tabular}

Table 2.4: Summary of assumptions in deterministic model

\subsection{Deterministic Model}

The deterministic model maximizes the system spinning and operating reserves considering the highest demand case or worst outage case. The main disadvantage of this approach is that it neglects the stochastic nature of the maintenance scheduling, such as the uncertainty of system demand, or forced outages of generators and transmission lines. Neglecting the randomness of maintenance scheduling may lead to a very high operating cost. Meanwhile, an unforeseen failure is possible to happen, which could affect the whole system. Table 2.4 shows different assumptions in deterministic models.

As we can see from Table 2.4, most of the deterministic models have the assumptions that: the power demand in each period is known and typically peak demand is chosen as the threshold for the demand constraint. However in reality, power demand is not all the same during one period, so using this deterministic demand may increase the generation cost. Another assumption is the prices of fuel, energy, and ancillary services are known; however the spot prices are fluctuating all the times, and neglecting the uncertainty in prices may lose money. The last assumption is that the forced outages of generators and transmission lines are 0 , this assumption could dramatically simplify the maintenance scheduling problem, but it may cause inaccuracy of the scheduling.

Chattopadhyay et al. [19] presented a Mixed Integer Programming (MIP) model considering fuel production, fuel transportation, maintenance scheduling, generation scheduling and inter-utility transfers. Coal production capacity constraint and coal linkage constraint are two different ones from others. The author compared the maintenance scheduling methods among Levelized Reserve, LOLP Levelling, LOLP minimization, and Least Cost method. 
They conclude that even though the Least Cost method has the highest LOLP, it is within a 0.05 permissible limit. Also, the effect of fuel supply constraints has been analysed in detail. Finally, the author confirmed that inter-area transfer consideration could significantly reduce the overall system costs.

Conejo et al. [16] explored the coordination between ISO and producers. Two models are introduced accordingly: maximizing reliability for ISO and maximizing profit for producers. In the plan for ISO, the target is to maximize the reliability index which is net reserve divided by gross reserve along the time horizon. A seven step detailed procedure is introduced in order to obtain a generation maintenance plan that could not only achieve a sufficient level of reliability, but also guarantee producers with maximum profits. It should be pointed out that in step 3, a tuned-up incentive/disincentive is considered to direct producers to modify their plan and achieve a sufficient level of reliability.

Escudero [18] aimed to find a strong lower bound for the solution of maintenance scheduling problems. It formulates the model as minimizing the operating cost over the planning horizon, subject to four types of constraints: one time maintenance, start-up logic, generation capacity and demand constraints. In order to reduce the difficulty caused by binary variables in non-linear problems, the last three constraints are relaxed by replacing them with some continuous constraints suggested by Biggs [34]. After transforming the original problem into a non-linear problem, it is solved through constrained non-linear programming algorithm.

Kim et al. [29] formulated the maintenance problem as a deterministic 0-1 integer programming. It assumes that the power demand for each period is known. The constraints include demand constraint, consecutive periods of maintenance, maintenance crew constrain$\mathrm{t}$, and available extensions. The difference from others is that the variance of spinning reserve rate is also considered in the objective function. In order to find a better solution, it combines Tabu search, Genetic Algorithm, and simulated annealing method to solve the problem.

Marwali and Shahidehpour [45] presented a Mixed Integer Programming (MIP) model to solve both generation and transmission maintenance scheduling problems simultaneously. The decoupling constraints (network constraints) represented by transportation model are the key parts in their paper. They use Benders decomposition to decompose the global 
generator/transmission scheduling problem into a master problem which minimizes unit maintenance and transmission maintenance cost subject to coupling constraints, and a subproblem considering minimizing generation cost subject to decoupling constraints.

\subsection{Stochastic Model}

The other category is the stochastic model. Many works have focused on how to simulate the maintenance scheduling, and transform a large scale stochastic model into a number of deterministic problems. In this way, a maintenance scheduling may ensure an acceptable risk level and maximize social welfare.

In this part, we would like to discuss different approaches incorporating the stochastic nature of maintenance scheduling problems among literatures, at the end of this part, we will show the comparison of assumptions.

- Stochastic load: electricity demand is one of the uncertainties and complexities of the maintenance scheduling problem. Typically, models considering the stochastic load usually are trying to minimize the total expected cost.

Often times, total expected cost is calculated summing up the costs which are considered, such as maintenance cost, generation cost, start-up cost, and so on, then multiply by the scenario probability. Following is an example which considers maintenance cost and generation cost,

$$
\min \sum_{s \in S} p_{s} *\left(M_{s}+G_{s}\right)
$$

where $s$ represents one power demand scenario in all the scenarios $S . M_{s}$ is the maintenance cost of scenario $s, G_{s}$ is the generation cost of scenario $s$.

There are many authors using strategies to simulate the uncertainty of electricity demand. For example, Kralj and Petrovic [23] modeled stochastic demand by off-peak and on-peak load duration curves (LDC). Gaussian probability distribution is used to calculate deviation between peak load and a forecasted value. They performed 
simulation before the optimization in order to save the computation during the searching procedure. They treated the maintenance scheduling problem as a combinatorial optimization task: minimizing fuel costs, maximizing reliability, and minimizing constraints violations simultaneously. Branch and bound is used to solve the problem.

Canto [31] formulated the maintenance scheduling problem into three demand scenarios: high, medium, and low electricity demand. The possibility of each scenario is fixed by each specific power plant, for example, in the case study, the possibility of electric demand of a 75 power plant grid is 0.1 ( low demand ), 0.6 ( medium demand ), 0.3 ( high demand ). Each scenario is divided into 13 months, then divided into 6 subperiods namely peak, middle, and low demand of business days and weekend days. By claiming that maintenance cost is insignificant regarding start-up and production cost, the author does not consider maintenance cost in the objective function. The model is clearly represented by maintenance constraints, economic unit commitment constraints, maintenance and connection constraints, and generating volume constraints. The problem is solved by Bender's decomposition.

- Uncertain forced outages of transmission lines and generating units: in Marwali and Shahidehpour [13], whether forced outages will happen is decided at the end of a week in the short term scheduling. Long term scheduling is determined by those forecasted parameters. The authors used a two-state continuous-time Markov model to calculate the forced outage rates of each utility. Then the updated value of forced outage rates are applied to the network constraints in the long term scheduling, Monte-Carlo sampling is used to generate 1000 random future scenarios, each of them represents the availability of generating units and transmission lines. Then these 1000 samples are evaluated by dynamic scheduling, using the system reserve energy as the indicator.

- Stochastic load and forced outages of generators and transmission lines: except for the stochastic nature of demand, the uncertainty in generation capacity and transmission line availability has also been considered. For example, Silva et al. [24] considered the uncertainties of generators, transmission lines, and peak load of certain areas defined by stochastic vectors. The operation subproblem aimed at minimizing the expected 
energy not served (EPNS). The operation subproblem is checked with a pre-established upper bound on EPNS in the original problem. First, the master problem (decision) establishes a trial maintenance schedule subjected to resource, sequence and other constraints. This result is one of the stochastic vectors. Then this vector is checked in the operation sub problem. If the trail schedule does not violate operation subproblem reliability constraint, the proposed trial schedule is optimal. Otherwise, Benders cut is generated and added to the master problem. This procedure is performed until convergence.

- Uncertainties in prices of energy, ancillary services, and fuel: from the GENCO's point of view, the goal is to maximize its payoffs and minimize its financial risk. The payoff of a GENCO is highly dependent on the prices of energy, ancillary services, and fuel. If a GENCO could adjust its maintenance outage to periods when the prices of energy and ancillary services are relatively low and the price of fuel is high, it could save a lot of money. In $\mathrm{Wu}$ et al. [14], the author realized this coordination by Monte Carlo simulation. First, a stochastic maintenance scheduling model which maximizes the GENCOS's payoff subject to maintenance outage windows and resource constraints is proposed, then it introduces hourly priced-based unit commitment constraints including energy, ancillary services, fuel constraints, and many other constraints. Following is the objective function which incorporates uncertain prices in scenarios,

$$
\max \sum_{s \in S} \sum_{t \in T} p_{s} *\left\{G_{t,(s)}+S_{t,(s)}+N S_{t,(s)}+B_{t,(s)}-\left(M_{t,(s)}+F_{t,(s)}+S D_{t,(s)}\right)\right\},
$$

where $G_{t,(s)}, S_{t,(s)}, N S_{t,(s)}, B_{t,(s)}$ represent generation income, spinning reserve income, non-spinning reserve income, and bilateral income of scenario $s$ in period $t, M_{t,(s)}, F_{t,(s)}$, $S D_{t,(s)}$ represent maintenance cost, fuel cost, and shut down cost of scenario $s$ in period $t$.

Monte carlo method is used to generate all initial scenarios, each of them has an associated probability. After checking the bundle constraints, each scenario subproblem is solved in order to maximize payoffs in the entire period. Once a trial schedule is obtained, fuel, emission, and maintenance coupling constraints are checked. If all the 
constraints are not violated, go to the next scenario, otherwise recalculate the problem by updating Lagrangian multipliers. After the solution for all the scenarios are found, an optimal schedule is obtained if the solution does not violate the bundle constraints among scenarios.

- Stochastic forced outages, fuel prices,and load forecast errors

In $\mathrm{Wu}$ et al. [15], Lagrangian relaxation is used to decompose the original problem, moreover, the Monte Carlo method is used to simulate the random outages of generators and transmission lines, fuel price fluctuations and load forecast errors. Scenario bundle constraints are decomposed in long term security-constrained UC subproblem, thus the final solution is a combination of weighted scenarios. The objective function is

$$
\min \sum_{s \in S} \sum_{t \in T}\left\{M_{t,(s)}+p_{s} *\left(F_{t,(s)}+S D_{t,(s)}+S U_{t,(s)}\right)+P_{t,(s)}\right\},
$$

where $S U_{t,(s)}, P_{t,(s)}$ represent start up cost and penalty cost of scenario $s$ in period $t$. As we showed before, the uncertainty of forced outages of generating units and transmission lines, the load forecast errors, the fluctuation in energy, ancillary services, and fuel prices are all key items considered in stochastic maintenance scheduling models. In Table 2.5, some authors choose one of them to deal with, some choose the combination of them. The key point in proposing a stochastic maintenance scheduling model is how to simulate different scenarios, each of them has a corresponding probability. Branch-and-bound and Monte-Carlo simulation are used to realize that goal. 


\begin{tabular}{|c|c|}
\hline Assumptions & References \\
\hline $\begin{array}{l}\text { Power demand in each time period is mod- } \\
\text { eled by load duration curves }\end{array}$ & $\begin{array}{l}{[25],[23],[46],[47],[24],[30],} \\
{[22],[48],[15]}\end{array}$ \\
\hline $\begin{array}{l}\text { The fluctuation in prices of fuel, energy, } \\
\text { and ancillary services could influence a } \\
\text { GENCO's payoff }\end{array}$ & {$[21],[14],[15]$} \\
\hline Forced outage rate is considered & $\begin{array}{l}{[46],[47],[30],[24],[30],[21],} \\
{[15]}\end{array}$ \\
\hline
\end{tabular}

Table 2.5: Summary of assumptions in stochastic model 


\section{Chapter 3}

\section{Power Plant Maintenance Considering Fuel Contracts}

In this chapter the assumptions of maintenance scheduling with gas portfolio have been made. A novel optimization model of maintenance scheduling is proposed including model reformulation and decomposition. Finally the computational results among the original model, reformulated model and decomposed model have been compared.

\subsection{Problem Statement}

This section introduces the logic and assumptions in the model. A Take-or-Pay gas portfolio is proposed. The rule of gas transfer between reservoirs and power plant has been defined.

\subsubsection{Maintenance Scheduling with Gas Portfolio}

In previous chapters we have introduced the maintenance within power plant itself. However the power plant is not isolated from the outside pool. All the market participants, such as GENCOS, TRANSCOS, and DISCOS, are closely related to power plants [49]. Henceforth many researchers have not only considered maintenance modeling within power plant, they have also took other participants into consideration. In this research, we assume that we 
are dealing with a power plant that runs on natural gas, a non-renewable resource. This assumption has motivated us to consider maintenance with gas portfolio because every natural gas power station needs to deal with gas purchasing.

Gas portfolio optimization is performed to optimally use the flexibility in gas purchasing [50]. It helps reduce the risks and costs in a gas portfolio when decision making is needed by managers, investors and traders. The uncertain demand is the first priority to be served. For example, in a power plant using natural gas generating electricity, the demand of gas is uncertain due to the uncertain demand of electricity from customers, maintenance of generating units, and so on. Then several aspects should be considered to meet the demand, such as gas purchasing price, gas spot market trading, and gas storage reservoir capacity. Gas portfolio optimization could contribute to the reduction of gas costs, and avoid buying gas from gas spot market at high price or not being able to meet demand.

The main goal of combining gas contract optimization and power plant maintenance scheduling is to decrease the cost for both purchasing gas and performing maintenance, and increase the revenue from power generation. Since the gas price and electricity price are fluctuating all the time, so these two factors are the main sources driving our model. Our goal is to purchase gas when gas price is relatively low, and postpone maintenance when electricity price is relatively high but not overuse the power generating units without maintenance.

\section{Take-or-Pay Gas Contract}

We consider a Take-or-Pay(ToP) gas contract in this project. A Take-or-Pay gas contract is a rule established between companies and gas suppliers [51]. The company must take an agreed amount of gas from the supplier [52]. We model the Take-or-pay gas contract with the maximum monthly gas withdraw volume of $M$, together with the minimum monthly withdraw percentage $X \%$. We also have an annual minimum withdraw percentage of $Y \%(Y \geq X)$, and maximum storage time of gas of $N$ periods. The following assumptions are made in this project:

- Two reservoirs are considered, A and B. Reservoir B is the receiver of yearly gas volume, and it can transfer gas to reservoir A in every month. Reservoir A is the receiver of 
monthly gas volume from both reservoir B and the distributors, and it can transfer gas to the power plant.

- Gas in reservoir A must be less than or equal to the maximum monthly gas withdraw volume of $M$, and greater than or equal to the minimum monthly gas withdraw volume of $X \% M$.

- At the beginning of each year, $Y \% * 12 M$ amount of gas is pushed into reservoir B.

- Reservoirs A and B must be restored to full capacity at the end of each year.

Figure 3.1 points out the gas transfer direction in our assumptions. It shows the ToP gas contract. If gas from the ToP contract cannot meet the demand of power plant, it will directly purchase gas from the spot market, and it can also sell the redundant gas to spot market.

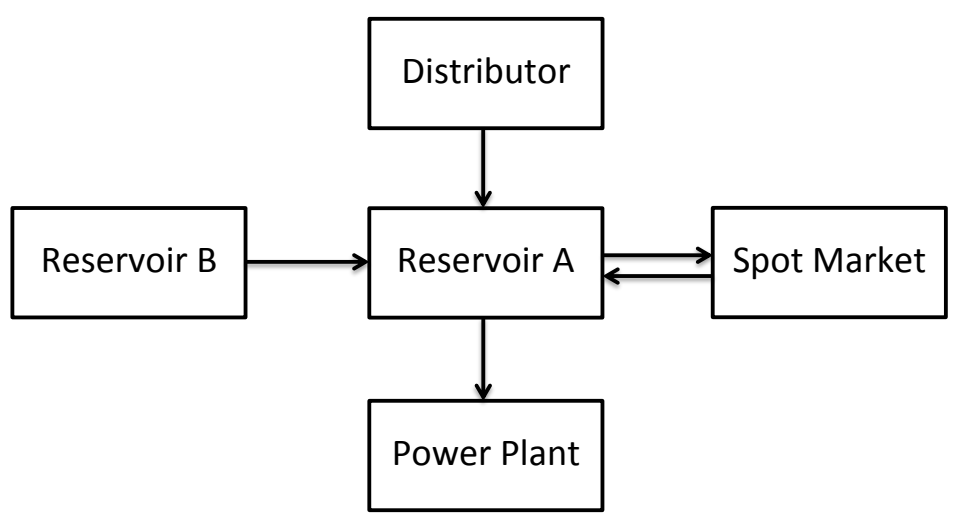

Figure 3.1: Gas transfer direction

\section{Maintenance Factors}

As we surveyed previously, there are a lot of factors one can consider in maintenance scheduling, such as maintenance duration, resource, priority, and so on. In this project, we consider three factors, namely maintenance duration, frequency, and maintenance cost. Table 3.1 shows an example of maintenance-related frequency, duration, and cost in a moderate-size power plant. 


\begin{tabular}{cccc}
\hline Equipment & Time between maintenance & Average Duration & Cost $(\$)$ \\
\hline \hline 1 & 2300 hours & 7 days & 1100 \\
2 & 2600 hours & 14 days & 1300 \\
3 & 2800 hours & 21 days & 1900 \\
\hline
\end{tabular}

Table 3.1: Maintenance factors

\subsubsection{The Assumptions}

To facilitate the model formulation, the mathematical model has the following assumptions:

1. Electricity generated can always be sold to the market.

2. The maximum storage time of gas in reservoirs A\&B is one year.

3. All maintenance performed has fixed amount of time and cost, and no contingency exist.

4. The gas usage per day of the power plant is a constant.

5. Gas availability is stable as long as it satisfies our gas contract logic.

\subsection{Stochastic Model}

Based on the assumptions and logic stated in previous section, this section introduces the original stochastic optimization model including objectives and constraints.

\subsubsection{Introduction to Stochastic Programming}

Compared with deterministic problems, stochastic problems take uncertainties into consideration [53]. The stochastic programming model is more suitable to describe the problems since unknown parameter exists in the real world. In stochastic programming models, uncertainty is expressed in probabilities. The series of events and actions which are possible to happen are called scenarios. Most of stochastic problems have probability distributions for 
scenarios. The optimization goal is to minimize or maximize the expectations of objective function [54].

A stage is a point of progress. Decisions must be made in each stage. In a two stage stochastic model, a set of decisions need to be made without full information on some random events [55]. These decisions are called first-stage decisions. The second-stage or corrective actions are taken when full information is received on the realization of some random factor [56].

The multistage stochastic programming problems is an extension of the two stage problems described above. A sequence of observations $\xi_{1}, \xi_{2}, \ldots, \xi_{t}$ will be used as decision making criterion. The current stage decisions are made based on previous observations. Figure 3.2 shows the multistage decision process[57].

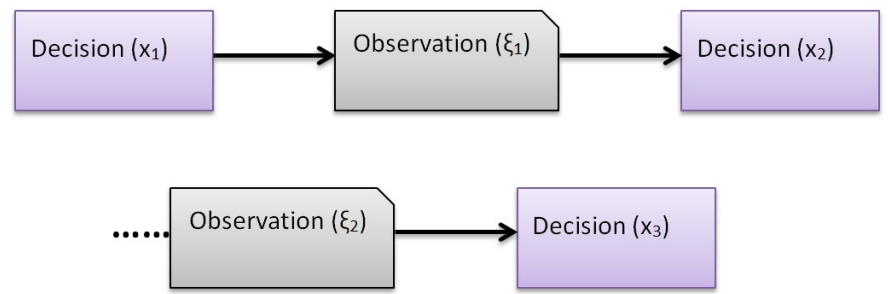

Figure 3.2: Multistage decision process

Let node 1 to be the root node. Since a decision needs to be made without full information when $t=1$, there is only one node in the first stage. After the first stage, if we assume that there are two uncertainties in each stage, then every node will generate two nodes in the following stage. Let $a(n)$ be the ancestor of node $n$. Figure 3.3 shows the scenario tree with three stages. If node $n$ is a leaf node, then the path from the root node to $n$ is called a scenario $(k)[58]$. Each scenario has a corresponding probability $\left(P_{k}\right)$.

In our model, multistage stochastic model is applied. Each month is considered as a stage. Gas and electricity price in each month will be the uncertainties which could influence the maintenance and power generation decisions. 

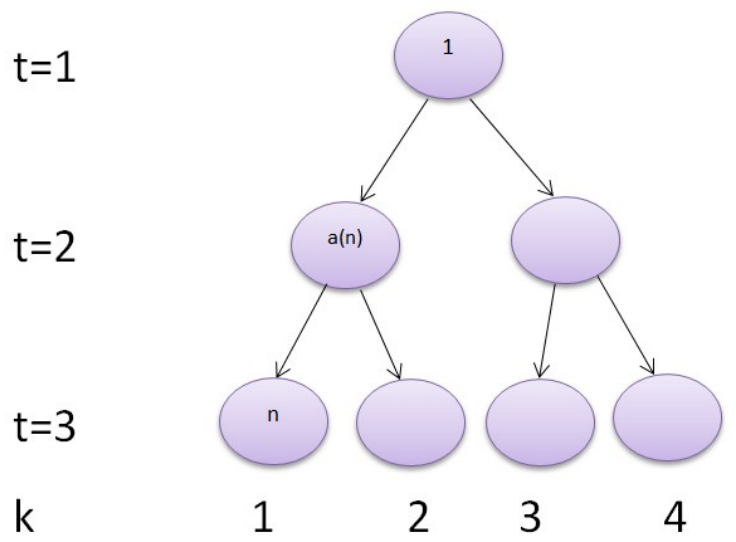

Figure 3.3: Scenario tree

\begin{tabular}{|c|l|}
\hline Indices & Descriptions \\
\hline$\xi$ & Senarios \\
$i$ & Maintenance equipments \\
$t$ & Time period(days, months or years) \\
$T$ & Time horizon to be considered(years) \\
$T_{0}$ & Beginning of each year \\
$T_{E}$ & End of each year \\
$n$ & Scenario bundles node \\
$j$ & columns \\
\hline
\end{tabular}

Table 3.2: Indices

\subsubsection{General Nomenclature}

The indices, parameters and variables used in the stochastic model are showed in Table $3.2,3.3,3.4$.

\subsubsection{Objective Function}

The objective of this study is to minimize negative of the total profit of electricity generation over the planning horizon. $\operatorname{prob}^{\xi}$ means the probability of the occurrence of each scenario $\xi ; c_{t}^{\xi}\left(m_{t}^{\xi}+f_{t}^{\xi}\right)$ is the gas purchasing cost from the contract, and $q_{t}^{\xi} s_{t}^{\xi^{-}}$is the gas purchasing cost from spot market; $h_{t}^{\xi}\left(g_{t}^{\xi}+s_{t}^{\xi^{-}}\right)$is the revenue coming from selling electricity, and $p_{t}^{\xi} s_{t}^{\xi^{+}}$is the income for selling redundant gas to spot market; $u_{i} z_{i, t}^{\xi}$ is the maintenance 


\begin{tabular}{|c|c|}
\hline Variables & Descriptions \\
\hline$a_{t}^{\xi}$ & the gas level in reservoir $\mathrm{A}$ at the end each month $\mathrm{t}$ (MMBTU) \\
\hline$b_{t}^{\xi}$ & the gas level in reservoir B at the end each month $t$ (MMBTU) \\
\hline$r_{i, t}^{\xi}$ & the remaining days before the next required maintenance \\
\hline & at the end of each month $t$ for equipment $i$ \\
\hline$g_{t}^{\xi}$ & the amount of gas used for generating electricity in each month $t$ (MMBTU) \\
\hline$m_{t}^{\xi}$ & $\begin{array}{l}\text { the amount of gas purchased from distributors for reservoir A } \\
\text { in each month } \mathrm{t} \text { (MMBTU) }\end{array}$ \\
\hline$f_{t}^{\xi}$ & $\begin{array}{l}\text { the amount of gas transferred from reservoir B to reservoir } A \\
\text { in each month } \mathrm{t} \text { (MMBTU) }\end{array}$ \\
\hline$s_{t}^{\xi^{+}}$ & the amount of gas sold to the spot market in each month $t$ (MMBTU) \\
\hline$s_{t}^{\xi^{-}}$ & the amount of gas bought from the spot market in each month $\mathrm{t}$ (MMBTU) \\
\hline$z_{i, t}^{\xi}$ & $\begin{array}{l}\text { binary variable denoting a decision of performing maintenance, such that } \\
z_{i, t}^{\xi}=1 \\
\text { if we schedule maintenance during month } t \text { for equipment } i, \\
\text { and } z_{i, t}^{\xi}=0 \text { otherwise }\end{array}$ \\
\hline
\end{tabular}

Table 3.3: Variables

\begin{tabular}{|c|c|}
\hline Parameters & Descriptions \\
\hline$c_{t}^{\xi}$ & gas contract price in each month $t$ (\$/MMBTU) \\
\hline$h_{t}^{\xi}$ & $\begin{array}{l}\text { electricity price worth per unit gas of scenario } \xi \text { in each month } t \\
(\$ / \text { MMBU })\end{array}$ \\
\hline$p_{t}^{\xi}$ & $\begin{array}{l}\text { gas price sold to the spot market of scenario } \xi \text { in each month } t(\$ / \mathrm{MMB}- \\
\text { TU) }\end{array}$ \\
\hline$q_{t}^{\xi}$ & $\begin{array}{l}\text { gas price purchased from the spot market of scenario } \xi \text { in each month } t \\
(\$ / \text { MMBT })\end{array}$ \\
\hline$u_{i}$ & maintenance cost for equipment $i(\$)$ \\
\hline$M$ & maximum monthly gas withdraw volume for reservoir A (MMBTU) \\
\hline$X \%$ & minimum monthly withdraw percentage for reservoir A \\
\hline$Y \%$ & annual minimum withdraw percentage for reservoir B \\
\hline$\Delta_{i}$ & maximal length of time for equipment $i$ without maintenance (days) \\
\hline$\sigma_{i}$ & maintenance duration for equipment $i$ (days) \\
\hline$C$ & gas usage per day (MMBTU/day) \\
\hline$\delta_{i, t}$ & time-in-use of equipment $i$ during month $t$ (days) \\
\hline
\end{tabular}

Table 3.4: Parameters 
cost. The total profit is the summation of the above items with income having a negative sign and outcome having a positive sign. The objective function can be represented as follows,

$$
\min \sum_{\xi \in \Xi} \operatorname{prob}^{\xi}\left[\sum_{t \in T}\left(c_{t}^{\xi}\left(m_{t}^{\xi}+f_{t}^{\xi}\right)-h_{t}^{\xi}\left(g_{t}^{\xi}+s_{t}^{\xi^{-}}\right)-p_{t}^{\xi} s_{t}^{\xi^{+}}+q_{t}^{\xi} s_{t}^{\xi^{-}}\right)+\sum_{i \in I} \sum_{t \in T} u_{i} z_{i, t}^{\xi}\right]
$$

\subsubsection{Constraints}

The motivation in this project is to consider both maintenance part and gas portfolio part, and then connect those two with their relationships. The motivation is represented by the following constraints.

\section{Gas Balance In Reservoirs}

In this model, we consider the gas balance in reservoir A and B between one month and the previous month. For reservoir A, the input is the amount of gas purchased from distributors $\left(m_{t}^{\xi}\right)$ and transferred from reservoir $\mathrm{B}\left(f_{t}^{\xi}\right)$, and the output will be the amount of gas sold to the spot market $\left(s_{t}^{\xi^{+}}\right)$and used for electricity generation $\left(g_{t}^{\xi}\right)$. For each scenario $\xi$ and each time period $t$, gas balance constraint can be represented as follows:

$$
a_{t}^{\xi}=a_{t-1}^{\xi}+m_{t}^{\xi}+f_{t}^{\xi}-s_{t}^{\xi^{+}}-g_{t}^{\xi}
$$

For reservoir $\mathrm{B}$, there will be only one output, that is the amount of gas transferred to reservoir $\mathrm{A}\left(f_{t}^{\xi}\right)$ :

$$
b_{t}^{\xi}=b_{t-1}^{\xi}-f_{t}^{\xi}
$$

\section{Gas Transfer Logic}

According to the assumption, for reservoir A, the maximum monthly gas withdraw volume is $M$, and the minimum gas withdraw percentage is $X \%$. Again, for each scenario $\xi$ and each time period $t$, gas transfer logic can be represented as follows:

$$
X \% M \leq m_{t}^{\xi}+f_{t}^{\xi} \leq M
$$


For reservoir $\mathrm{B}$, the annual minimum withdraw percentage is $Y \%$ :

$$
b_{t}^{\xi}=Y \%(12 M)-f_{t}^{\xi}
$$

Also, reservoir A and B must be restored to full capacity at the end of the maximum storage time $N$, and if $N$ is one year, then the following constraints are established:

$$
b_{t}^{\xi}=0, a_{t}^{\xi}=0
$$

when $t=12$.

\section{Maintenance Logic}

At the end of each month $t$, the remaining days before the next required maintenance $\left(r_{i, t}^{\xi}\right)$ should be related with the maintenance decision $\left(z_{i, t}^{\xi}\right)$ and electricity generation $\left(\delta_{i, t}\right)$ in each month $t$. Maintenance logic for each scenario $\xi$, each equipment $i$ and each time period $t$ can be represented as follows:

$$
r_{i, t}^{\xi} \leq r_{i, t-1}^{\xi}-\delta_{i, t}+\left(\Delta^{i}+\sigma^{i}\right) z_{i, t}^{\xi}
$$

The upper bound of $r_{i, t}^{\xi}$ should be the maximum days without maintenance minus the usage of equipment $i$ in each month $t$ :

$$
r_{i, t}^{\xi} \leq \Delta^{i}+\sigma^{i}-\delta_{i, t}
$$

\section{Power Generation Logic}

The amount of gas used for electricity generation in each month $t$ should be related with the maintenance decision. The lower number of maintenance happened in one month, the higher amount of gas would be consumed to generate electricity:

$$
g_{t}^{\xi}+s_{t}^{\xi^{-}} \leq C\left(\delta_{i, t}-\sigma^{i} z_{i, t}^{\xi}\right)
$$




\section{Nonanticipativity Constraint}

We use $S_{n, t}$ to denote scenario bundles node $n$ if scenario $\xi$ and $\eta$ have the same history until time $t$ at node $n$, so variables related with node $n$ should have the same decisions in time $t$ :

$$
x_{t}^{\xi}=x_{t}^{\eta}, \quad \forall(\xi, \eta) \in S_{n, t}
$$

where $x_{t}^{\xi}=\left[a_{t}^{\xi}, b_{t}^{\xi}, g_{t}^{\xi}, s_{t}^{\xi^{+}}, s_{t}^{\xi^{-}}, m_{t}^{\xi}, f_{t}^{\xi}, z_{t}^{\xi}, r_{t}^{\xi}\right]$. 


\subsubsection{Optimization Model}

The general stochastic programming model is summarized as follows:

$$
\begin{aligned}
& \min \sum_{\xi \in \Xi} \operatorname{prob}^{\xi}\left[\sum_{t \in T}\left(c_{t}^{\xi}\left(m_{t}^{\xi}+f_{t}^{\xi}\right)-h_{t}^{\xi}\left(g_{t}^{\xi}+s_{t}^{\xi^{-}}\right)-p_{t}^{\xi} s_{t}^{\xi^{+}}+q_{t}^{\xi} s_{t}^{\xi^{-}}\right)+\sum_{i \in I} \sum_{t \in T} u_{i} z_{i, t}^{\xi}\right] \\
& \text { s.t. } \quad a_{t}^{\xi}=a_{t-1}^{\xi}+m_{t}^{\xi}+f_{t}^{\xi}-s_{t}^{\xi^{+}}-g_{t}^{\xi}, \quad \forall t \in T, \xi \in \Xi \text {, } \\
& b_{t}^{\xi}=b_{t-1}^{\xi}-f_{t}^{\xi}, \quad \forall t \in T \backslash T_{0}, \xi \in \Xi, \\
& X \% M \leq m_{t}^{\xi}+f_{t}^{\xi} \leq M, \quad \forall t \in T, \xi \in \Xi, \\
& b_{t}^{\xi}=Y \%(12 M)-f_{t}^{\xi}, \quad \forall t \in T_{0}, \xi \in \Xi, \\
& b_{t}^{\xi}=0, a_{t}^{\xi}=0, \quad \forall t \in T_{E}, \xi \in \Xi \text {, } \\
& r_{i, t}^{\xi} \leq r_{i, t-1}^{\xi}-\delta_{i, t}+\left(\Delta_{i}+\sigma_{i}\right) z_{i, t}^{\xi}, \forall t \in T \backslash 1, \xi \in \Xi, i \in I, \\
& g_{t}^{\xi}+s_{t}^{\xi^{-}} \leq C\left(\delta_{i, t}-\sigma_{i} z_{i, t}^{\xi}\right), \quad \forall t \in T, \xi \in \Xi, i \in I, \\
& r_{i, t}^{\xi} \leq \Delta_{i}+\sigma_{i}-\delta_{i, t}, \quad \forall t \in T, \xi \in \Xi, i \in I, \\
& x_{t}^{\xi}=x_{t}^{\eta}, \quad \forall(\xi, \eta) \in S_{n, t}, t \in T, n \in N, \xi \in \Xi, \\
& z_{i, t}^{\xi} \in\{0,1\}, \quad \forall t \in T, i \in I, \xi \in \Xi, \\
& a_{t}^{\xi}, b_{t}^{\xi}, g_{t}^{\xi}, s_{t}^{\xi^{+}}, s_{t}^{\xi^{-}}, m_{t}^{\xi}, f_{t}^{\xi}, r_{i, t}^{\xi} \geq 0 \forall t \in T, i \in I, \xi \in \Xi,
\end{aligned}
$$

where $x_{t}^{\xi}=\left[a_{t}^{\xi}, b_{t}^{\xi}, g_{t}^{\xi}, s_{t}^{\xi^{+}}, s_{t}^{\xi^{-}}, m_{t}^{\xi}, f_{t}^{\xi}, z_{t}^{\xi}, r_{t}^{\xi}\right], \quad z_{t}^{\xi}=\left[z_{1, t}^{\xi}, \ldots, z_{i, t}^{\xi}\right], r_{t}^{\xi}=\left[r_{1, t}^{\xi}, \ldots, r_{i, t}^{\xi}\right]$. $\xi$ denotes scenarios. Constraint (3.2) and (3.3) show the balance of gas level in reservoir $A$ and $B$ in each period. Constraint (3.4) points out the upper and lower bound of monthly withdraw volume. Constraint (3.10) is the nonanticipativity constraint.

\subsection{Model Reformulation}

We can interpret maintenance in the way that maintenance in one period must be performed so that the decreasing of capacity of the equipment can be restored. In this way, maintenance is considered as replenishment to the usage of the equipment. We can also consider that the maintenance in one period will affect the following periods such as the maintenance decisions in those periods. In other words, maintenance is considered as an action which will impact the following periods. If maintenance is interpreted in the second 
way, we need to answer how it affect the following periods and what is the contribution of one maintenance in terms of meeting the capacity requirements in the following periods.

The main contribution of reformulation is obtaining a tighter approximation of the convex hull of feasible solutions so that the computational speed will be faster [59]. We use $\zeta_{\tau t}^{i}$ to denote whether maintenance of $i$ in period $\tau$ contributes toward meeting the capacity requirement of $\mathrm{i}$ in period $t, \phi_{i}$ denotes equipment $i$ should be maintained every $\phi$ months, and $\tau$ denotes time periods (days, months, or years).

Figure 3.4 shows that if one equipment must been maintained every three months, then we can split maintenance decision variable in time period $3\left(z_{3}\right)$ into three variables: $\zeta_{13}, \zeta_{23}, \zeta_{33}$. Instead of analyzing $z_{3}$, we are now interested in what will affect $z_{3}$ using $\zeta_{13}, \zeta_{23}, \zeta_{33}$.

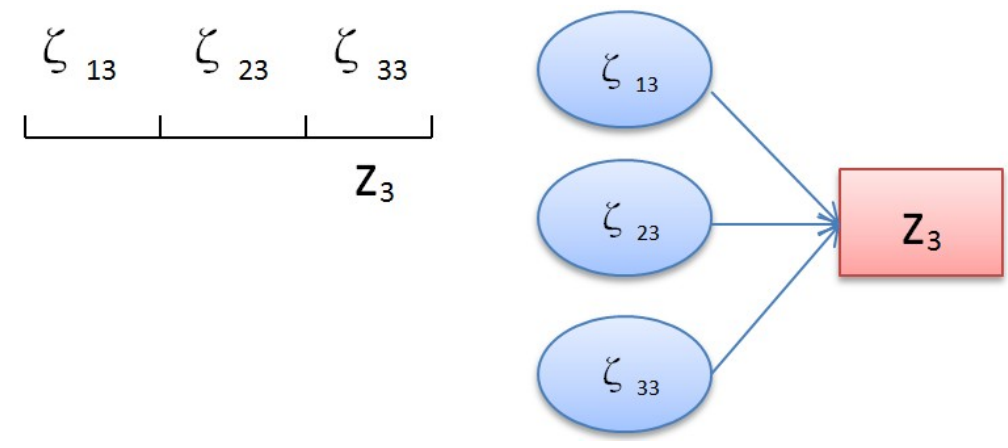

Figure 3.4: Reformulation technique

According to [59], if the summation of $\zeta_{\tau t}^{i}$ is greater or equal to 1 which means at least there will be one $\zeta_{\tau t}^{i}$ contribute toward meeting the capacity requirement in the following $\tau$ periods, the equipment will be in good condition since all the necessary maintenance has been performed. So constraint (3.7)\&(3.9) can be reformulated as

$$
\begin{aligned}
& \zeta_{t-\tau, t} \leq z_{t-\tau}, \quad \forall \tau \in\left[0, \phi_{i}-1\right] \\
& \sum_{\tau=0}^{\phi_{i}-1} \zeta_{t-\tau, t} \geq 1
\end{aligned}
$$

According to the previous principles, the original stochastic programming model can be developed to a reformulated model as follows: 


$$
\begin{aligned}
& \min \sum_{\xi \in \Xi} \operatorname{prob}^{\xi}\left[\sum_{t \in T}\left(c_{t}^{\xi}\left(m_{t}^{\xi}+f_{t}^{\xi}\right)-h_{t}^{\xi}\left(g_{t}^{\xi}+s_{t}^{\xi^{-}}\right)-p_{t}^{\xi} s_{t}^{\xi^{+}}+q_{t}^{\xi} s_{t}^{\xi^{-}}+\sum_{i \in I} \sum_{t \in T} u_{i} z_{i, t}^{\xi}\right](\right. \\
& \text { s.t. } \quad a_{t}^{\xi}=a_{t-1}^{\xi}+m_{t}^{\xi}+f_{t}^{\xi}-s_{t}^{\xi^{+}}-g_{t}^{\xi}, \quad \forall t \in T, \xi \in \Xi \text {, } \\
& b_{t}^{\xi}=b_{t-1}^{\xi}-f_{t}^{\xi}, \quad \forall t \in T \backslash T_{0}, \xi \in \Xi, \\
& X \% M \leq m_{t}^{\xi}+f_{t}^{\xi} \leq M, \quad \forall t \in T, \xi \in \Xi, \\
& b_{t}^{\xi}=Y \%(12 M)-f_{t}^{\xi}, \quad \forall t \in T_{0}, \xi \in \Xi, \\
& b_{t}^{\xi}=0, a_{t}^{\xi}=0, \quad \forall t \in T_{E}, \xi \in \Xi \\
& \zeta_{i, t-\tau, t}^{\xi} \leq z_{i, t-\tau}^{\xi}, \quad \forall \tau \in\left[0, \phi_{i}-1\right], t \in T, \xi \in \Xi, i \in I, \\
& \sum_{\tau=0}^{\phi_{i}-1} \zeta_{i, t-\tau, t}^{\xi} \geq 1, \quad \forall t \in T, \xi \in \Xi, i \in I, \\
& g_{t}^{\xi}+s_{t}^{\xi^{-}} \leq C\left(\delta_{i, t}-\sigma_{i} z_{i, t}^{\xi}\right), \quad \forall t \in T, \xi \in \Xi, i \in I, \\
& x_{t}^{\xi}=x_{t}^{\eta}, \quad \forall(\xi, \eta) \in S_{n, t}, t \in T, n \in N, \xi \in \Xi, \\
& \zeta_{i, t-\tau, t}^{\xi} \in\{0,1\}, \quad \forall \tau \in\left[0, \phi_{i}-1\right], t \in T, \xi \in \Xi, i \in I, \\
& 0 \leq z_{i, t}^{\xi} \leq 1, \quad \forall t \in T, \xi \in \Xi, i \in I, \\
& a_{t}^{\xi}, b_{t}^{\xi}, g_{t}^{\xi}, s_{t}^{\xi^{+}}, s_{t}^{\xi^{-}}, m_{t}^{\xi}, f_{t}^{\xi} \geq 0 \quad \forall t \in T, \xi \in \Xi
\end{aligned}
$$

\subsection{Decomposition}

If we enlarge the problem, there will be a bottleneck that the computer cannot solve the model due to insufficient memory since there are too many feasible solutions for the computer to find all of them. In this case, decomposition could help solve large-scaled problems. The main idea of decomposition is that starting solving the model when some of the feasible solutions have been obtained serving as basis, then use this basis to search for more feasible solutions. When no more feasible solution can help improve the objective value, the combination of current feasible solutions is optimal. We use Dantzig-Wolfe Decomposition technique to further decompose the reformulated model [59].

Figure 3.5 shows the Dantzig-Wolfe Decomposition procedure. The master problem is solved initially, and the solution and dual are extracted. Then the subproblem is solved 


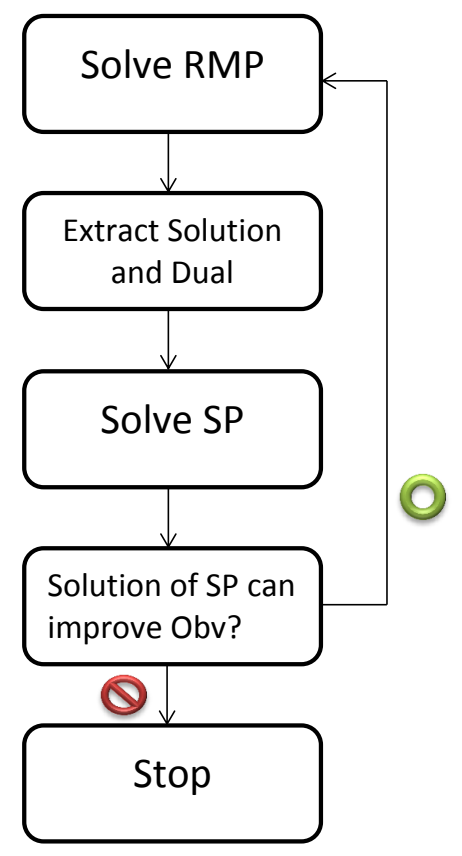

Figure 3.5: Decomposition

using the solution and dual extracted by the master problem. If the current solution of the subproblem can help improve the objective value, then the current solution of the subproblem is added to the master problem as a column, otherwise stop [60].

We use $j$ to denote columns in the master problem, and $\lambda_{j}^{\xi}$ denotes the weight of extreme points of scenario $\xi . \pi_{x_{t}^{\xi}}$ is the dual for the nonanticipativity constraint of variable $x_{t}^{\xi}$, and $\pi_{0}$ is the dual for the constraint of variable $\lambda_{j}^{\xi}$.

Compared with the reformulated model, we move the nonanticipitivity constraint into the master problem since we assume that it is the nonanticipitivity constraint which brings the complexity. The subproblem will be solved independently for every scenario, then we add the solution of the subproblem into the master problem as columns. The master problem has only two constraints. Solving the master problem can give us the dual value which will affect the solution of the subproblem in next loop. Based on these theories, the reformulated model can be decomposed as follows: 
SB-MP:

$$
\begin{aligned}
& \min \quad \sum_{\xi \in \Xi} \operatorname{prob}^{\xi} \sum_{j \in J_{\xi}} \hat{R}_{j}^{\xi} \lambda_{j}^{\xi} \\
& \text { s.t. } W_{x_{n}}-\sum_{j \in J_{\xi}}\left(\hat{x}_{t}^{\xi}\right)^{j} \lambda_{j}^{\xi}=0, \quad \forall(\xi, t) \in S_{n}, n \in N, \quad\left[\pi_{x_{t}^{\xi}}\right] \\
& \sum_{j \in J_{\xi}} \lambda_{j}^{\xi}=1, \quad \forall \xi \in \Xi, \quad\left[\pi_{0}^{\xi}\right] \\
& 0 \leq \lambda_{j}^{\xi} \leq 1, \quad \forall j \in J_{\xi}, \xi \in \Xi
\end{aligned}
$$

where $\left(\hat{x}_{t}^{\xi}\right)^{j}=\left[\left(\hat{a}_{t}^{\xi}\right)^{j},\left(\hat{b}_{t}^{\xi}\right)^{j},\left(\hat{m}_{t}^{\xi}\right)^{j},\left(\hat{f}_{t}^{\xi}\right)^{j},\left(\hat{g}_{t}^{\xi}\right)^{j},\left(\hat{s}_{t}^{\xi+}\right)^{j},\left(\hat{s}_{t}^{\xi-}\right)^{j},\left(\hat{z}_{t}^{\xi}\right)^{j}\right],\left(\hat{z}_{t}^{\xi}\right)^{j}=\left[\left(\hat{z}_{1, t}^{\xi}\right)^{j}, \ldots,\left(\hat{z}_{i, t}^{\xi}\right)^{j}\right]$, $\hat{R}_{j}^{\xi}=\sum_{t \in T}\left[\hat{c}_{t}^{\xi}\left(\left(\hat{m}_{t}^{\xi}\right)^{j}+\left(\hat{f}_{t}^{\xi}\right)^{j}\right)-\hat{h}_{t}^{\xi}\left(\left(\hat{g}_{t}^{\xi}\right)^{j}+\left(\hat{s}_{t}^{\xi-}\right)^{j}\right)-\hat{p}_{t}^{\xi}\left(\hat{s}_{t}^{\xi+}\right)^{j}+\hat{q}_{t}^{\xi}\left(\hat{s}_{t}^{\xi-}\right)^{j}\right]+\sum_{t \in T} \sum_{i \in I} \hat{u}_{i}\left(\hat{z}_{i, t}^{\xi}\right)^{j}$. $\operatorname{SB}-\operatorname{SP}(\xi)$ :

$$
\begin{aligned}
& \min \sum_{t \in T}\left[\left(c_{t}^{\xi}+\hat{\pi}_{m_{t}^{\xi}}\right) m_{t}^{\xi}+\left(c_{t}^{\xi}+\hat{\pi}_{f_{t}^{\xi}}\right) f_{t}^{\xi}-\left(h_{t}^{\xi}-\hat{\pi}_{g_{t}^{\xi}}\right) g_{t}^{\xi}-\left(h_{t}^{\xi}-\hat{\pi}_{s_{t}^{\xi-}}\right) s_{t}^{\xi-}\right. \\
& \left.-\left(p_{t}^{\xi}-\hat{\pi}_{s_{t}^{\xi+}}\right) s_{t}^{\xi+}+\left(q_{t}^{\xi}+\hat{\pi}_{s_{t}^{\xi-}}\right) s_{t}^{\xi-}+\hat{\pi}_{a_{t}^{\xi}} a_{t}^{\xi}+\hat{\pi}_{b_{t}^{\xi}} b_{t}^{\xi}\right] \\
& +\sum_{t \in T} \sum_{i \in I}\left(u_{i}+\hat{\pi}_{z_{i, t}^{\xi}}\right) z_{i, t}^{\xi}-\pi_{0}^{\xi} \\
& \text { s.t. } \quad a_{t}^{\xi}=a_{t-1}^{\xi}+m_{t}^{\xi}+f_{t}^{\xi}-s_{t}^{\xi^{+}}-g_{t}^{\xi}, \quad \forall t \in T \text {, } \\
& b_{t}^{\xi}=b_{t-1}^{\xi}-f_{t}^{\xi}, \quad \forall t \in T \backslash T_{0}, \\
& X \% M \leq m_{t}^{\xi}+f_{t}^{\xi} \leq M, \quad \forall t \in T, \\
& b_{t}^{\xi}=Y \%(12 M)-f_{t}^{\xi}, \quad \forall t \in T_{0}, \\
& b_{t}^{\xi}=0, a_{t}^{\xi}=0, \quad \forall t \in T_{E}, \\
& \zeta_{i, t-\tau, t}^{\xi} \leq z_{i, t-\tau}^{\xi}, \quad \forall \tau \in\left[0, \phi_{i}-1\right], t \in T, i \in I, \\
& \sum_{\tau=0}^{\phi_{i}-1} \zeta_{i, t-\tau, t}^{\xi} \geq 1, \quad \forall t \in T, i \in I, \\
& g_{t}^{\xi}+s_{t}^{\xi^{-}} \leq C\left(\delta_{i, t}-\sigma^{i} z_{i, t}^{\xi}\right), \quad \forall t \in T, i \in I, \\
& \zeta_{i, t-\tau, t}^{\xi} \in\{0,1\}, \quad \forall \tau \in\left[0, \phi_{i}-1\right], t \in T, i \in I, \\
& 0 \leq z_{i, t}^{\xi} \leq 1, \quad \forall t \in T, i \in I, \\
& a_{t}^{\xi}, b_{t}^{\xi}, g_{t}^{\xi}, s_{t}^{\xi^{+}}, s_{t}^{\xi^{-}}, m_{t}^{\xi}, f_{t}^{\xi} \geq 0, \quad \forall t \in T, i \in I .
\end{aligned}
$$




\subsection{Computational Results}

In this chapter, the solution of the original stochastic model is firstly analyzed. Then we compare the solution of the reformulated model with the original one to see whether they have the same result. Thirdly, the contribution of decomposition is discussed.

\subsubsection{Analyze the Solution of the Original Model}

We initially obtained all the rough data on Energy Information Administration. In order to show the effectiveness of the original model, we would like to see if we manipulate the data such as making the data higher in the first 6 months and lower in the following 6 months, the model will certainly give us logical solutions according to that manipulation. For this purpose, we manipulated $c_{t}^{\xi}, q_{t}^{\xi}$ and $h_{t}^{\xi}$ higher/lower in the first half of the year and lower/higher in the rest of the year, and we kept $p_{t}^{\xi}$ to be a very low value since we do not want to purchase any redundant gas and sold them to the spot market. The parameters used are as follows:

\begin{tabular}{l|llllllllllll}
\hline $\begin{array}{l}\text { Time } \\
\text { Period }\end{array}$ & 1 & 2 & 3 & 4 & 5 & 6 & 7 & 8 & 9 & 10 & 11 & 12 \\
\hline$c_{t}^{\xi}$ & 2.12 & 2.13 & 2.21 & 2.31 & 2.05 & 2.07 & 2.93 & 2.92 & 2.73 & 2.99 & 2.89 & 2.88 \\
$q_{t}^{\xi}$ & 2.66 & 2.76 & 2.73 & 2.63 & 2.83 & 2.70 & 2.32 & 2.31 & 2.28 & 2.15 & 2.22 & 2.18 \\
$h_{t}^{\xi}$ & 11.83 & 11.79 & 11.71 & 11.89 & 11.76 & 11.86 & 9.95 & 9.96 & 9.88 & 9.89 & 9.91 & 9.92 \\
$p_{t}^{\xi}$ & 1 & 1 & 1 & 1 & 1 & 1 & 1 & 1 & 1 & 1 & 1 & 1 \\
\hline
\end{tabular}

Table 3.5: Input parameters of prices $(\$ / M M B T U)$

\begin{tabular}{|c|c|c|c|c|}
\hline Scenario\# & Unit\# & $\Delta_{i}($ day $)$ & $\sigma_{i}($ day $)$ & $u_{i}(\$)$ \\
\hline 1 & 1 & 87 & 7 & 1000 \\
\hline
\end{tabular}

Table 3.6: Other parameters

The model was solved by IBM Cplex Optimization Studio. The objective value and solution reported by Cplex are as follows:

The maintenance decision reflects that the equipment must be maintained at least every 3 months which makes sense because we used 87 days as the longest time of one unit without 


\section{\begin{tabular}{l|l|l|}
\hline Objective Value & -481717 \\
\hline
\end{tabular}}

Table 3.7: Objective value of the original model using parameters in Table $3.5 \& 3.6$

\begin{tabular}{l|llllllllllll}
\hline $\begin{array}{l}\text { Time } \\
\text { Period }\end{array}$ & 1 & 2 & 3 & 4 & 5 & 6 & 7 & 8 & 9 & 10 & 11 & 12 \\
\hline$m_{t}^{\xi}$ & 0 & 5000 & 5000 & 5000 & 0 & 0 & 0 & 1000 & 0 & 1000 & 1000 & 0 \\
$f_{t}^{\xi}$ & 5000 & 0 & 0 & 0 & 5000 & 5000 & 1000 & 0 & 1000 & 0 & 0 & \\
$s_{t}^{\xi-}$ & 200 & 0 & 0 & 0 & 0 & 0 & 2120 & 2910 & 4100 & 5100 & 1910 & 4100 \\
$g_{t}^{\xi}$ & 4900 & 5100 & 3910 & 5100 & 3910 & 5100 & 2980 & 1000 & 1000 & 0 & 2000 & 1000 \\
$s_{t}^{\xi+}$ & 0 & 0 & 0 & 0 & 0 & 0 & 0 & 0 & 0 & 0 & 0 & 0 \\
$z_{i, t}^{\xi}$ & 0 & 0 & 1 & -0 & 1 & -0 & 0 & 1 & 0 & 0 & 1 & -0 \\
$r_{i, t}^{\xi}$ & 57 & 27 & 30 & 0 & 64 & 34 & 4 & 60 & 30 & 0 & 30 & 0 \\
$a_{t}^{\xi}$ & 100 & 0 & 1090 & 990 & 2080 & 1980 & 0 & 0 & 0 & 1000 & 0 & 0 \\
$b_{t}^{\xi}$ & 13000 & 13000 & 13000 & 13000 & 8000 & 3000 & 2000 & 2000 & 1000 & 1000 & 1000 & 0 \\
\hline
\end{tabular}

Table 3.8: Solution of the original model using parameters in Table 3.5 \& 3.6

maintenance. Also, we can interpret from the solution that the model is able to select the optimal gas sources, that is, when gas price in ToP contract is low, then ToP is selected as the gas source, when gas price from the distributor is low, then the distributor will provide gas. Finally, the electricity generation is significantly affected by the electricity price. For example, the amount of electricity generated in the first six months is much higher than the second half of the year due to the relatively high electricity price. Also, no gas has been sold to the spot market because the spot market selling price is very low. So model can responde to different input data and the solution makes sense.

\subsubsection{Compare the Solution and Computational Time of the O- riginal and Reformulated Model}

\section{Compare the Solution}

It is clear from Table 3.7 and 3.9 that the objective value of the reformulated model is exactly the same as the original one, and the solutions of the reformulated model are slightly different with the original ones since alternative solutions may exit. This indicates that 


\section{Objective Value -481717}

Table 3.9: Objective value of the reformulated model using parameters in Table 3.5 \& 3.6

\begin{tabular}{l|llllllllllll}
\hline $\begin{array}{l}\text { Time } \\
\text { Period }\end{array}$ & 1 & 2 & 3 & 4 & 5 & 6 & 7 & 8 & 9 & 10 & 11 & 12 \\
\hline$m_{t}^{\xi}$ & 0 & 0 & 0 & 5000 & 5000 & 5000 & 0 & 1000 & 0 & 0 & 1000 & 1000 \\
$f_{t}^{\xi}$ & 5000 & 5000 & 5000 & 0 & 0 & 0 & 1000 & 0 & 1000 & 1000 & 0 & 0 \\
$s_{t}^{\xi-}$ & 200 & 0 & 0 & 0 & 0 & 0 & 2120 & 2910 & 4100 & 5100 & 1910 & 4100 \\
$g_{t}^{\xi}$ & 4900 & 5100 & 3910 & 5100 & 3910 & 5100 & 2980 & 1000 & 1000 & 0 & 2000 & 1000 \\
$s_{t}^{\xi+}$ & 0 & 0 & 0 & 0 & 0 & 0 & 0 & 0 & 0 & 0 & 0 & 0 \\
$z_{i, t}^{\xi}$ & 0 & -0 & 1 & 0 & 1 & -0 & 0 & 1 & 0 & 0 & 1 & 0 \\
$a_{t}^{\xi}$ & 100 & 0 & 1090 & 990 & 2080 & 1980 & 0 & 0 & 0 & 1000 & 0 & 0 \\
$b_{t}^{\xi}$ & 13000 & 8000 & 3000 & 3000 & 3000 & 3000 & 2000 & 2000 & 1000 & 0 & 0 & 0 \\
\hline
\end{tabular}

Table 3.10: Solution of the reformulated model using parameters in Table $3.5 \& 3.6$

reformulation is successful because it gives the same or alternative solutions as the original model. 


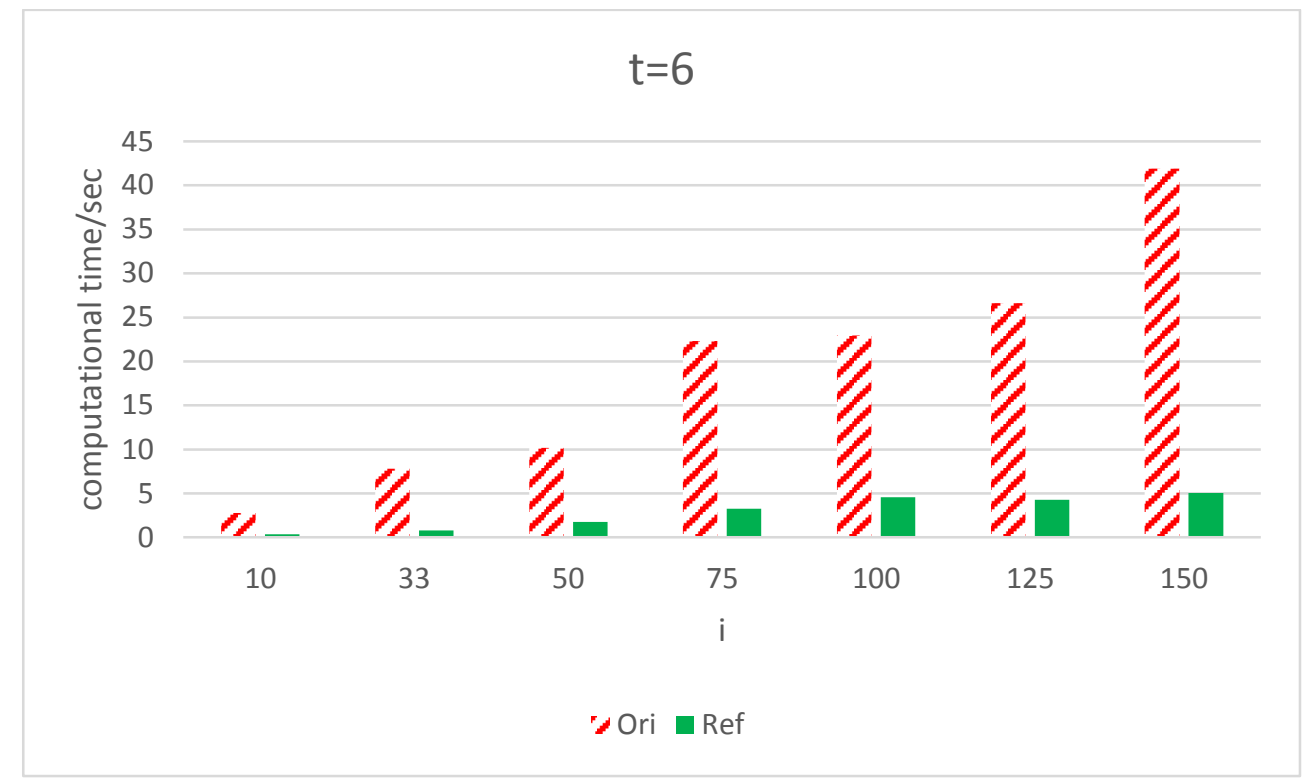

Figure 3.6: Compare computational time when $\mathrm{t}=6$

\section{Compare the Computational Time}

Except for the effectiveness of the reformulation, we are more interested with the efficiency. For this purpose, we continuously increased the number of period $t$ and the number of equipment $i$. We assumed that there will be 2 uncertainties in each time period, and the random data are generated in Excel based on the rough data obtained on Energy Information Administration. For example, if we consider 6 periods, the number of scenarios would be $2^{5}=32$. Figure 3.6, 3.7, and 3.8 compare the computational time between the original and reformulated models when we consider different time horizon and different number of equipment.

The reformulated model is very efficient when the time horizon considered is relatively short and the number of equipment is low. For example, when $t=9$, and $i=33$, the computational time of reformulation is only 17 seconds, while the original model needs 150 seconds. However, when we consider long time horizon and large number of equipment, the efficiency will drop. This is one part of the future research, that is, making the reformulated model 


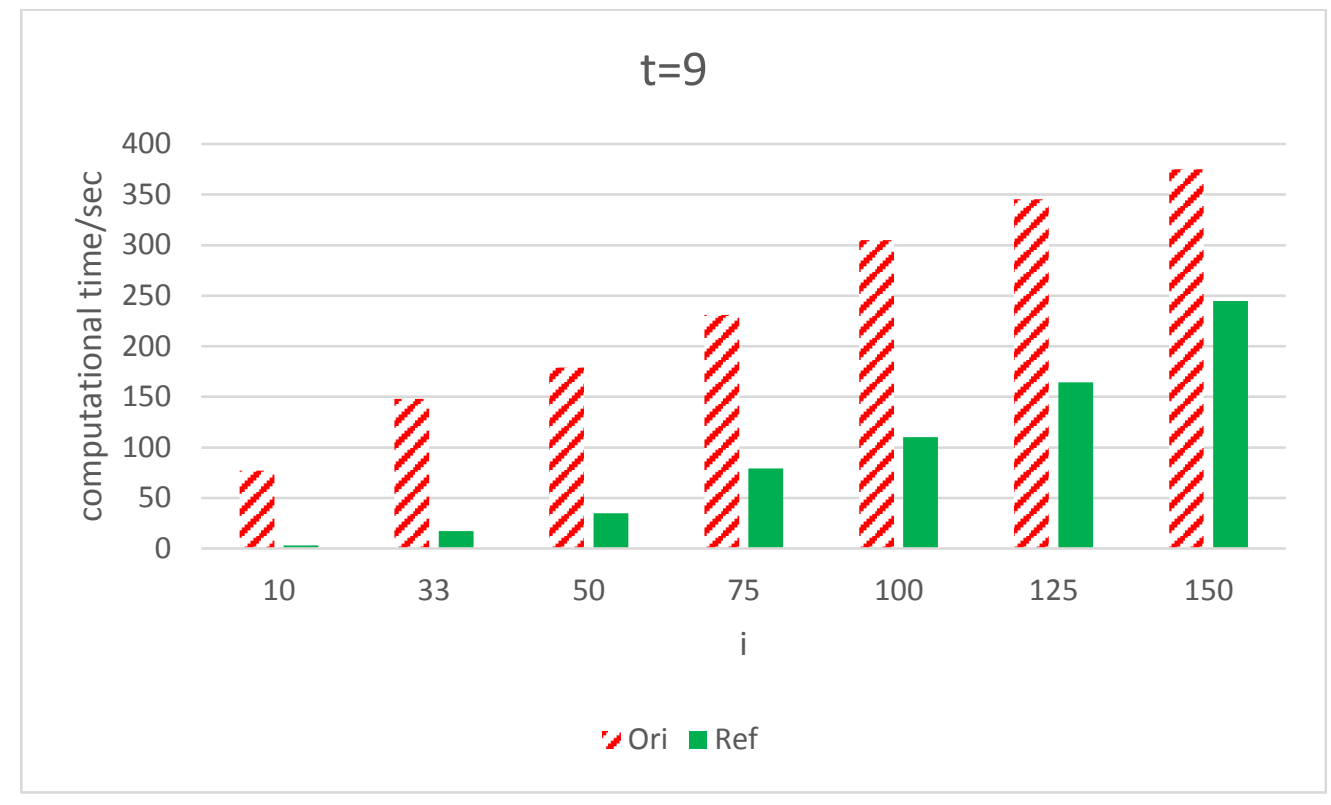

Figure 3.7: Compare computational time when $t=9$

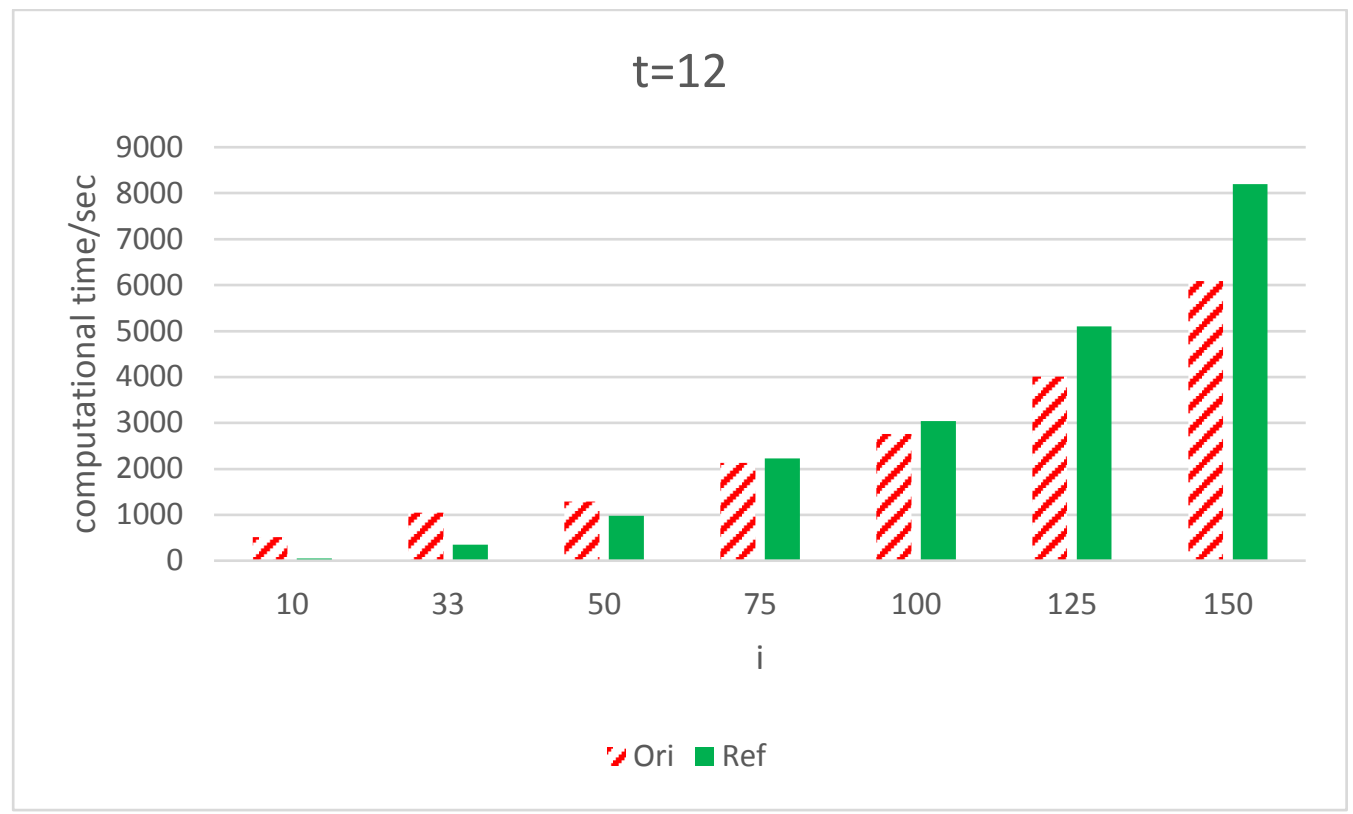

Figure 3.8: Compare computational time when $\mathrm{t}=12$ 
efficient when dealing with large-scale problems.

\subsubsection{Solution of the Decomposed Model}

We have selected number of time periods being 6 and number of scenarios being 32. Since $W_{z_{n}}$ is the maintenance decision in the decomposed model, the evolution of $W_{z_{n}}$ during loops will indicate how decomposition works. The solution of $W_{z_{n}}$ has been extracted and showed in Table 3.11 and 3.12:

In the first loop, all the $W_{z_{n}}$ are binary and the objective value is -250237 . The objective value continued decreasing as the number of loops increases. In the 22nd loop, the objective value is -257575 and $W_{z_{n}}$ became decimal. After the 29 th loop, the objective value remained -257946 and nearly all the $W_{z_{n}}$ are binary solutions with only four $W_{z_{n}}$ being decimals. This is the best solution our decomposed model can obtain so far. What is expected from the decomposed model is that the upper bond and lower bond should converge quickly and all the $W_{z_{n}}$ are binary when the objective value remains the same and cannot be further improved. This is also part of the future research.

\subsection{Conclusions and Future Research}

As we surveyed in Chapter 2, there are many kinds of decisions, constraints, and objectives used in previous literature of power plant maintenance scheduling. We established the assumption that we are dealing with the power plant using natural gas as resources, so gas portfolio should be considered in the model. Based on that assumption, least-cost based criterion is selected as objective since our primary goal is to minimize maintenance and production cost, as well as maximizing revenues. Also, a couple of constraints are established specifying maintenance logic, power generation logic, gas purchasing, and the relationship among those. Some variables are defined to represent maintenance decision, power generation, and gas reservoir status. The model is considered as a stochastic one since the possible combinations of data have been used as input.

The computational result of the original stochastic model indicates that if the input data has some general pattern, the model will return logical solutions according to the data. For 
example, the model would tell the decision maker where gas should be purchased and how much should be purchased according to gas price of different resources such as spot market and gas contract, the model also tells that how much electricity should be generated in each period. The most important aspect is that the model ensures that all the maintenance requirements are satisfied. The optimal objective value indicates how much profit will be generated under current data. However, computational speed of the original model is not very satisfying which motivated us to find better model formulations.

Maintenance problems can be considered as capacity planning problems. One reformulation technique has been successfully applied on capacity planning problems by splitting the capacity expansion variables into multiple ones. The benefit is obtaining a tighter convex hull which could help reduce computational time. We applied this reformulation technique on our maintenance decision variables, trying to analyze the effect of maintenance by asking what does one maintenance mean and how will it affect the following maintenances, in other words, what is the contribution of one maintenance in terms of meeting the capacity requirements in the following periods. The computational results of the reformulated model is satisfying because the reformulation not only gives us the optimal solution but also takes much less computational time. But the reformulation also has some bottleneck. The advantage of reformulation becomes less obvious as we expand the problem. This called us to propose decomposition.

When problem size is so large that no matter how powerful the computer is, the problem still cannot be solved by Cplex and it even cannot return one feasible solution because there are too many feasible solutions for Cplex to find all of them. Decomposition has some advantages in this situation. The main idea of decomposition is to only consider part of the feasible solutions. When part of the feasible solutions have been found, decomposition will add more feasible solutions through loops under the assumption that those added feasible solutions can help improve the objective value. Otherwise, when no further feasible solution can improve the objective value, the process will be terminated and we claim that the combination of current feasible solutions is optimal. Now this algorithm has already been achieved in Cplex: from the example computational results of decomposition we can see that the objective value has been improved continuously as more and more columns are added 
into the model. But we did not obtain the optimal solution since some of the maintenance decision variables are not binary. As we discussed before, decomposition starts from some basic feasible solutions. In order to find the optimal solution of decomposition, we may improve the original feasible solutions added to the model serving as basis by either changing the number of feasible solutions added at the beginning of decomposition or changing the data used to obtain those original feasible solutions.

Since the reformulated model is efficient only when the problem size is small. We did not obtain the optimal solution from the decomposed model, and the converging speed is very slow. Future work will focus on the following areas:

- Accelerate the computational speed of the reformulated model, enable it to solve largescale problems very quickly.

- Accelerate the converging speed of the decomposed model so that the optimal solution can be obtained in a reasonable time.

- The proposed optimization model will be evaluated by comparing computational results with production schedules obtained in the real world. 


\begin{tabular}{|c|c|c|c|c|c|c|c|}
\hline Loop & 1 & 22 & 29 & Loop & 1 & 22 & 29 \\
\hline Wz000 & 0 & 0 & 0 & Wr1016 & 0 & 0 & 0 \\
\hline Wz001 & 0 & 0 & 0 & Wz1017 & 0 & 0 & 0 \\
\hline Wz002 & 0 & 0 & 0 & Wz1018 & 0 & 0 & 0 \\
\hline Wz003 & 0 & 0 & 0 & Wz1019 & 0 & 0 & 0 \\
\hline Wz004 & 0 & 0 & 0 & Wz1020 & 0 & 0 & 0 \\
\hline Wz005 & 0 & 0 & 0 & Wz1021 & 0 & 0 & 0 \\
\hline Wz006 & 0 & 0 & 0 & Wz1022 & 0 & 0 & 0 \\
\hline Wz007 & 0 & 0 & 0 & Wz1023 & 0 & 0 & 0 \\
\hline Wz008 & 0 & 0 & 0 & Wz1024 & 0 & 0 & 0 \\
\hline Wz009 & 0 & 0 & 0 & Wz1025 & 0 & 0 & 0 \\
\hline Wz0010 & 0 & 0 & 0 & Wz1026 & 0 & 0 & 0 \\
\hline Wz0011 & 0 & 0 & 0 & Wz1027 & 0 & 0 & 0 \\
\hline Wz0012 & 0 & 0 & 0 & Wz1028 & 0 & 0 & 0 \\
\hline Wz0013 & 0 & 0 & 0 & Wz1029 & 0 & 0 & 0 \\
\hline Wz0014 & 0 & 0 & 0 & Wz1030 & 0 & 0 & 0 \\
\hline Wz0015 & 0 & 0 & 0 & Wz1031 & 0 & 0 & 0 \\
\hline Wz0016 & 0 & 0 & 0 & Wz200 & 1 & 0.946329 & 1 \\
\hline Wz0017 & 0 & 0 & 0 & Wz201 & 1 & 0.946329 & 1 \\
\hline Wz0018 & 0 & 0 & 0 & Wz202 & 1 & 0.946329 & 1 \\
\hline Wz0019 & 0 & 0 & 0 & Wz203 & 1 & 0.946329 & 1 \\
\hline Wz0020 & 0 & 0 & 0 & Wz204 & 1 & 0.946329 & 1 \\
\hline Wz0021 & 0 & 0 & 0 & Wz205 & 1 & 0.946329 & 1 \\
\hline Wz0022 & 0 & 0 & 0 & Wz206 & 1 & 0.946329 & 1 \\
\hline Wz0023 & 0 & 0 & 0 & Wz207 & 1 & 0.946329 & 1 \\
\hline Wz0024 & 0 & 0 & 0 & Wz208 & 1 & 0.946329 & 1 \\
\hline Wz0025 & 0 & 0 & 0 & Wz209 & 1 & 0.946329 & 1 \\
\hline Wz0026 & 0 & 0 & 0 & Wz2010 & 1 & 0.946329 & 1 \\
\hline Wz0027 & 0 & 0 & 0 & Wz2011 & 1 & 0.946329 & 1 \\
\hline Wz0028 & 0 & 0 & 0 & Wz2012 & 1 & 0.946329 & 1 \\
\hline Wz0029 & 0 & 0 & 0 & Wz2013 & 1 & 0.946329 & 1 \\
\hline Wz0030 & 0 & 0 & 0 & Wz2014 & 1 & 0.946329 & 1 \\
\hline Wz0031 & 0 & 0 & 0 & Wz2015 & 1 & 0.946329 & 1 \\
\hline Wz100 & 0 & 0.0536712 & 0 & Wz2016 & 1 & 1 & 1 \\
\hline Wz101 & 0 & 0.0536712 & 0 & Wz2017 & 1 & 1 & 1 \\
\hline Wz102 & 0 & 0.0536712 & 0 & Wz2018 & 1 & 1 & 1 \\
\hline Wz103 & 0 & 0.0536712 & 0 & Wz2019 & 1 & 1 & 1 \\
\hline Wz104 & 0 & 0.0536712 & 0 & Wz2020 & 1 & 1 & 1 \\
\hline Wz105 & 0 & 0.0536712 & 0 & Wz2021 & 1 & 1 & 1 \\
\hline Wz106 & 0 & 0.0536712 & 0 & Wz2022 & 1 & 1 & 1 \\
\hline Wz107 & 0 & 0.0536712 & 0 & Wz2023 & 1 & 1 & 1 \\
\hline Wz108 & 0 & 0.0536712 & 0 & Wz2024 & 1 & 1 & 1 \\
\hline Wz109 & 0 & 0.0536712 & 0 & Wz2025 & 1 & 1 & 1 \\
\hline Wz1010 & 0 & 0.0536712 & 0 & Wz2026 & 1 & 1 & 1 \\
\hline Wz1011 & 0 & 0.0536712 & 0 & Wz2027 & 1 & 1 & 1 \\
\hline Wz1012 & 0 & 0.0536712 & 0 & Wz2028 & 1 & 1 & 1 \\
\hline Wz1013 & 0 & 0.0536712 & 0 & Wz2029 & 1 & 1 & 1 \\
\hline Wz1014 & 0 & 0.0536712 & 0 & Wz2030 & 1 & 1 & 1 \\
\hline Wz1015 & 0 & 0.0536712 & 0 & Wz2031 & 1 & 1 & 1 \\
\hline
\end{tabular}

Table 3.11: Solution of $W_{z_{n}}$ of decomposed model, Part 1 


\begin{tabular}{|c|c|c|c|c|c|c|c|}
\hline Loop & 1 & 22 & 29 & Loop & 1 & 22 & 29 \\
\hline Wz300 & 0 & 0.0665487 & 0 & Wz4016 & 1 & 0 & 0 \\
\hline Wz301 & 0 & 0.0665487 & 0 & Wz4017 & 1 & 0 & 0 \\
\hline Wz302 & 0 & 0.0665487 & 0 & Wz4018 & 1 & 0 & 0 \\
\hline Wz303 & 0 & 0.0665487 & 0 & Wz4019 & 1 & 0 & 0 \\
\hline Wz304 & 0 & 0.0631072 & 0.00423958 & Wz4020 & 1 & 0 & 0 \\
\hline Wz305 & 0 & 0.0631072 & 0.00423958 & Wz4021 & 1 & 0 & 0 \\
\hline Wz306 & 0 & 0.0631072 & 0.00423958 & Wz4022 & 1 & 0 & 0 \\
\hline Wz307 & 0 & 0.0631072 & 0.00423958 & Wz4023 & 1 & 0 & 0 \\
\hline Wz308 & 0 & 0.0536712 & 0 & Wz4024 & 1 & 0 & 0 \\
\hline Wz309 & 0 & 0.0536712 & 0 & Wz4025 & 1 & 0 & 0 \\
\hline Wz3010 & 0 & 0.0536712 & 0 & Wz4026 & 1 & 0 & 0 \\
\hline Wz3011 & 0 & 0.0536712 & 0 & Wz4027 & 1 & 0 & 0 \\
\hline Wz3012 & 0 & 0.0536712 & 0 & Wz4028 & 1 & 0 & 0 \\
\hline Wz3013 & 0 & 0.0536712 & 0 & Wz4029 & 1 & 0 & 0 \\
\hline Wz3014 & 0 & 0.0536712 & 0 & Wz4030 & 1 & 0 & 0 \\
\hline Wz3015 & 0 & 0.0536712 & 0 & Wz4031 & 1 & 0 & 0 \\
\hline Wz3016 & 0 & 0 & 0 & & & & \\
\hline Wz3017 & 0 & 0 & 0 & & & & \\
\hline Wz3018 & 0 & 0 & 0 & & & & \\
\hline Wz3019 & 0 & 0 & 0 & & & & \\
\hline Wz3020 & 0 & 0 & 0 & & & & \\
\hline Wz3021 & 0 & 0 & 0 & & & & \\
\hline Wz3022 & 0 & 0 & 0 & & & & \\
\hline Wz3023 & 0 & 0 & 0 & & & & \\
\hline Wz3024 & 0 & 0 & 0 & & & & \\
\hline Wz3025 & 0 & 0 & 0 & & & & \\
\hline Wz3026 & 0 & 0 & 0 & & & & \\
\hline Wz3027 & 0 & 0 & 0 & & & & \\
\hline Wz3028 & 0 & 0 & 0 & & & & \\
\hline Wz3029 & 0 & 0 & 0 & & & & \\
\hline Wz3030 & 0 & 0 & 0 & & & & \\
\hline Wz3031 & 0 & 0 & 0 & & & & \\
\hline Wz400 & 1 & 0.0248519 & 0 & & & & \\
\hline Wz401 & 1 & 0.0248519 & 0 & & & & \\
\hline Wz402 & 1 & 0.0758763 & 0 & & & & \\
\hline Wz403 & 1 & 0.0758763 & 0 & & & & \\
\hline Wz404 & 1 & 0.00560909 & 0 & & & & \\
\hline Wz405 & 1 & 0.00560909 & 0 & & & & \\
\hline Wz406 & 1 & 0.0176817 & 0 & & & & \\
\hline Wz407 & 1 & 0.0176817 & 0 & & & & \\
\hline Wz408 & 1 & 0.0639356 & 0 & & & & \\
\hline Wz409 & 1 & 0.0639356 & 0 & & & & \\
\hline Wz4010 & 1 & 0.0438035 & 0 & & & & \\
\hline Wz4011 & 1 & 0.0438035 & 0 & & & & \\
\hline Wz4012 & 1 & 0.0903802 & 0 & & & & \\
\hline Wz4013 & 1 & 0.0903802 & 0 & & & & \\
\hline Wz4014 & 1 & 0.0347284 & 0 & & & & \\
\hline Wz4015 & 1 & 0.0347284 & 0 & & & & \\
\hline
\end{tabular}

Table 3.12: Solution of $W_{z_{n}}$ of decomposed model, Part 2 


\section{References}

[1] "Annual energy review 2011," Tech. Rep., U.S. Energy Information Administration, September 2012.

[2] J. J. Conti, P. D. Holtberg, J. A. Beamon, S. A. Napolitano, A. M. Schaal, and J. T. Turnure, "Annual energy outlook 2012," Tech. Rep., U.S. Energy Information Administration, June 2012.

[3] "Electric power annual 2011," Tech. Rep., U.S. Energy Information Administration, January 2013.

[4] C. H. Lie and Y. H. Chun, "An algorithm for preventive maintenance policy," Reliability, IEEE Transactions on, vol. 35, no. 1, pp. 71-75, April.

[5] M. Shahidehpour, H. Yamin, and Z. Li, Market operations in electric power systems:Forecasting, Scheduling, and Risk Management, New York:Wiley, 2002.

[6] Q. P. Zheng, J. Wang, P. M. Pardalos, and Y. Guan, "A decomposition approach to the two-stage stochastic unit commitment problem," Annals of Operations Research, vol. 210, pp. 387-410, November 2013.

[7] Y. Fu, M. Shahidehpour, and Z. Li, "Ac contingency dispatch based on securityconstrained unit commitment," Power Systems, IEEE Transactions on, vol. 21, no. 2, pp. 897-908, 2006.

[8] D. Dentcheva and W. Römisch, "Optimal power generation under uncertainty via stochastic programming," in Stochastic Programming Methods and Technical Applications (K. Marti and P. Kall Eds.), Lecture Notes in Economics and Mathematical Systems. 1997, pp. 22-56, Springer-Verlag.

[9] B. F. Hobbs, M. H. Rothkopf, R. P. ONeil, and H. Chao, The next generation of electric power unit commitment models, Norwell: Kluwer Academic, 2001.

[10] P. Carpentier, G. Gohen, J. C. Culioli, and A. Renaud, "Stochastic optimization of unit commitment: a new decomposition framework," Power Systems, IEEE Transactions on, vol. 11, no. 2, pp. 1067-1073, 1996. 
[11] L. Wu and M. Shahidehpour, "Accelerating the benders decomposition for networkconstrained unit commitment problems," Energy Systems, vol. 1, no. 3, pp. 339-376, August 2010.

[12] R. V. Canfield, "Cost optimization of periodic preventive maintenance," Reliability, IEEE Transactions on, vol. 35, no. 1, pp. 78-81, April.

[13] M. K. C. Marwali and S. M. Shahidehpour, "Coordination between long-term and short-term generation scheduling with network constraints," IEEE Transactions on power systems, vol. 15, no. 3, pp. 1161-1167, August 2000.

[14] L. Wu, M. Shahidehpour, and T. Li, "Genco's risk-based maintenance outage scheduling," IEEE Transactions on power systems, vol. 23, no. 1, pp. 127-136, February 2008.

[15] L. Wu, M. Shahidehpour, and Y. Fu, "Security-constrained generation and transmission outage scheduling with uncertainties," IEEE Transaction on power systems, vol. 25, no. 3, pp. 1674-1685, August 2010.

[16] A. J. Conejo, R. Garcia-Bertrand, and M. Diaz-Salazar, "Generation maintenance scheduling in restructed power systems," IEEE Transaction on power plant systems, vol. 20, no. 2, pp. 984-992, May 2005.

[17] J. Yellen, T. M. Al-Khamais, S. Vemuri, and L. Lemonidis, "A decomposition approach to unit maintenance scheduling," Transactions on Power systems, vol. 7, no. 2, pp. 726-733, May 1992.

[18] L. F. Escudero, "Lower bounds strategies in combinatorial nonlinear programming. a case study: Energy generators maintenance and operation scheduling," Qestii, vol. 5, no. 2, pp. 85-93, June 1981.

[19] D. Chattopadhyay, K. Bhattacharya, and J. Parikh, "A systems approach to least-cost maintenance scheduling for an interconnected power system," IEEE Transactions on Power Systems, vol. 10, no. 4, pp. 2002-2007, November 1995.

[20] D. Hayashi, K. Nagasaka, and C. H. K. Chu, "Generation maintenance scheduling for deregulated power systems," International Conference on Electrical Engineering 2002, 2002.

[21] T. Geetha and K. S. Swarup, "Coordinated maintenance scheduling of gencos and transcos in restructured power system," 2006.

[22] Y. Fu, M. Shahidehpour, and Z. Li, "Security-constrained optimal coordination of generation and transmission maintenance outage scheduling," IEEE Transaction on power plant systems, vol. 22, no. 3, pp. 1302-1313, August 2007.

[23] B. Kralj and R. Petrovic, "A multiobjective optimization approach to thermal generating units maintenance scheduling," European Journal of Operational Research, vol. 84, pp. 481-493, September 1995. 
[24] E. L. Silva, M. Morozowski, L. G. D. Fonseca, G. C. Oliveira, A. C. G. Melo, and J. C. O. Mello, "Transmission constrained maintenance scheduling of generating units: A stocastic programming approcach," IEEE Transactions on Power Systems, vol. 10, no. 2, pp. 695-701, May 1995.

[25] K. W. Edwin and F. Curtius, "New maintenance scheduling method with production cost minimization via integer linear programming," International journal of electrical power energy systems, vol. 12, pp. 165-170, July 1990.

[26] T. Satoh and K. Nara, "Maintenance scheduling by using simulated annealing method," IEEE Transactions on Power Systems, vol. 6, no. 2, pp. 850-857, May 1991.

[27] J. F. Dopazo and H. M. Merrill, "Optimal generator maintenance scheduling using integer programming," IEEE Transactions on Power Apparatus and Systems, vol. PAS94, no. 5, pp. 1537-1545, September/October 1975.

[28] R. Mukerji and J. H. Parker, "Power plant maintenance scheduling: optimizing economics and reliability," IEEE Transactions on Power Systems, vol. 6, no. 2, pp. 476-473, May 1991.

[29] H. Kim, Y. Hayashi, and K. Nara, "An algorithm for thermal unit maintenance scheduling through combined use of ga sa and ts," IEEE Transactions on Power systems, vol. 12, no. 1, pp. 329-335, February 1997.

[30] E. L. da Silva, M. Th. Schilling, and M. C. Rafael, "Generation maintenance scheduling consideration transmission constraints," IEEE Transaction on Power Systems, vol. 15, no. 2, pp. 838-843, May 2000.

[31] S. P. Canto, "Application of bender's decomposition to power plant preventive maintenance scheduling," European Journal of Operational Research, vol. 184, pp. 759-777, January 2008.

[32] C. A. Koay and Srinivasan, "Particle swarm optimization-based approach for generator maintenance scheduling," in Swarm Intelligence Symposium, 2003 SIS 03, Proceedings of the 2003 IEEE, 2003, pp. 167-173.

[33] L. Wu, M. Shahidehpour, and T. Li, "Cost of reliability analysis based on stochastic unit commitment," IEEE Transaction on power plant systems, vol. 23, no. 3, pp. 1364-1374, August 2008.

[34] C. Biggs, An Approach to Optimal Scheduling of an Electrical Power System, Technical report: Numerical Optimisation Centre. Numerical Optimisation Centre, Hatfield Polytechnic, 1975.

[35] L. Krishnasamy, F. Khan, and M. Haddara, "Development of a resk-based maintenance(rbm) strategy for a power-generating plant," Science Direct, vol. 18, pp. 69-81, January 2005. 
[36] F. G. Carazas and G. F. M. Souza, "Risk-based decision making method for maintenance policy selection of thermal power plant equipment," Energy, vol. 35, no. 2, pp. $964-$ $975,2010$.

[37] A. K. Nayak, D. K. Mohanta, and M. J. B. Reddy, "Generation reliability evaluation incorporating maintenance scheduling and load forecasting," in Environment and Electrical Engineering (EEEIC), 2012 11th International Conference on, may 2012, pp. 485 -489 .

[38] G. Bretthauer, T. Gamaleja, E. Handschin, U. Neumann, and W. Hoffmann, "Integrated maintenance scheduling system for electrical energy systems," Power Delivery, IEEE Transactions on, vol. 13, no. 2, pp. 655-660, April 1998.

[39] H. H. Zürn and V. H. Quintana, "Generaor maintenance scheduling via successive approximations dynamic programming," Power Apparatus and Systems, IEEE Transactions on, vol. 94, no. 2, pp. 665-671, March 1975.

[40] L. Chen and Junichi Toyoda, "Optimal generating unit maintenance scheduling for multi-area system with network constraints," Transactions on Power Systems, vol. 6, no. 3, pp. 1168-1174, August 1991.

[41] W. K. Foong, H. R. Maier, and A. R. Simpson, "Ant colony optimization for power plant maintenance scheduling optimization," Annals of Operations Research, vol. 159, pp. 433-450, June 2005.

[42] D. K. Mohanta, P. K. Sadhu, and R. Chakrabarti, "Fuzzy reliability evaluation of captive power plant maintenance scheduling incorporating uncertain forced outage rate and load representation," Science Direct, vol. 72, pp. 73-84, November 2004.

[43] D. K. Mohanta, P. K. Sadhu, and R. Chakrabarti, "Deterministic and stochastic approach for safety and reliability optimization of captive power maintenance scheduling using ga/sa-based hybrid techniques: A comparison of results," Science Direct, vol. 92, pp. 187-199, 2007.

[44] Q. P. Zheng, S. Rebennack, P. M. Pardalos, N. A. Iliadis, and M. V. F. Pereira, Handbook of CO2 in Power Systems, Springer, April 2012.

[45] M. K. C. Marwali and S. M. Shahidehpour, "A deterministic approach to generation and transmission maintenance scheduling with network constraints," Electric Power Systems Research, vol. 47, pp. 101-113, March 1998.

[46] M. K. C. Marwali and S. M. Shahidehpour, "Integrated generation and transmission maintenance scheduling with network constraints," IEEE Transactions on Power systems, vol. 13, no. 3, pp. 1063-1068, August 1998.

[47] M. K. C. Marwali and S. M. Shahidehpour, "Long-term transmission and generation maintenance scheduling with network, fuel and emission constraints," IEEE Transactions on Power Systems, vol. 14, no. 3, pp. 1160-1165, August 1999. 
[48] Y. Fu, Z. Li, M. Shahidehpour, T. Zheng, and E. Litvinov, "Coordination of midter$\mathrm{m}$ outage scheduling with short-term security-constrained unit commitment," IEEE Transaction on power plant systems, vol. 24, no. 4, pp. 1818-1830, November 2009.

[49] M. Shahidehpour H. Yamin, S. AI-Agtash, "Security-constrained optimal generation scheduling for gencos," IEEE, vol. 19, pp. 1365-1372, 2004.

[50] D. P. Fichter, "Application of genetic algorithms in portfolio optimization for the oil and gas industry," SPE Annula Technical Conference and Exhibition, 2000.

[51] S. E. Masten and K. J. Crocker, "Efficient adaptation in long-term contracts: Take-orpay provisions for natural gas," The American Economic Review, vol. 75, pp. 1083-1093, 1985.

[52] Q. P. Zheng, S. Rebennack, N. A. Iliadis, and P. M. Pardalos, "Optimization models in natural gas industry," Handbook of Power Systems I, pp. 121-148, 2010, Springer.

[53] Q. P. Zheng and P. M. Pardalos, "Stochastic and risk management models and solution algorithm for gas transmission network expansion and lng terminal location planning," Journal of Optimization Theory and Applications, vol. 147, pp. 337-357, 2010.

[54] Y. Huang, S. Rebennack, and Q. P. Zheng, "Techno-economic analysis and optimization models for carbon capture and storage - a survey.," Energy Systems, vol. 4, no. 4, pp. 315-353, December 2013.

[55] Y. Huang, Q. P. Zheng, and J. Wang, "Two-stage stochastic unit commitment model including non-generation resources with conditional value-at-risk constraints," Electrical Power Systems Research, in press, 2014.

[56] A. Shapiro and A. Kleywegt, "Minimax analysis of stochastic problems," Optimization Methods and Software, vol. 17, pp. 523-542, 2010.

[57] Y. Huang, Q. P. Zheng, N. Fan, and K. Aminian, "Optimal scheduling for enhanced coal bed methane production through co2 injection," Applied Energy, vol. 113, pp. 1475-1483, January 2014.

[58] H. Heitsch and W. Romisch, "Scenario tree modeling for multistage stochastic programs," Mathematical Programming, vol. 118, pp. 371-406, 2009.

[59] K. J. Singh, A. B. Philpott, and R. K. Wood, "Dantzig-wolfe decomposition for solving multistage stochastic capacity-planning problems," Operation Research, vol. 57, pp. 1271-1286, 2009.

[60] F. Vanderbeck, "On dantzig-wolfe decomposition in integer programming and ways to perform branching in a branch-and-price algorithm," Operations Research, vol. 48, pp. 111-128, 2000. 